\title{
Article \\ Compositional and Morphological Investigations of Roman Glass from Cremation Deposits at Birdoswald Fort on Hadrian's Wall, UK
}

\author{
Francesca Gherardi (D)
}

check for updates

Citation: Gherardi, F. Compositional and Morphological Investigations of Roman Glass from Cremation

Deposits at Birdoswald Fort on

Hadrian's Wall, UK. Heritage 2022, 5 , 362-377. https://doi.org/10.3390/ heritage5010021

Academic Editors: Massimo Lazzari

Received: 13 December 2021

Accepted: 8 February 2022

Published: 9 February 2022

Publisher's Note: MDPI stays neutral with regard to jurisdictional claims in published maps and institutional affiliations.

Copyright: (C) 2022 by the author. Licensee MDPI, Basel, Switzerland. This article is an open access article distributed under the terms and conditions of the Creative Commons Attribution (CC BY) license (https:/ / creativecommons.org/licenses/by/ $4.0 /)$.
Investigative Science, Historic England, Fort Cumberland Laboratories, Portsmouth, Hampshire P04 9LD, UK; francesca.gherardi@historicengland.org.uk

\begin{abstract}
Several different types of burial were identified during the excavation of the Roman military cemetery associated with the fort at Birdoswald, on Hadrian's Wall (UK). Fragments of glass vessels and glass beads were recovered from many of the cremation deposits, as they were commonly used during cremation rituals, and many of these had been affected by heat. X-ray fluorescence spectroscopy and scanning electron microscopy were used to investigate the raw materials, colorants and opacifiers employed to produce the glass assemblage. Most of the large fragments are transparent with a blue-green colour, with a composition typical of recycled glass. The smaller fragments are from beads and are coloured and sometimes opaque. Colourants and opacifiers characteristic of Roman glass were added in this glass formulation, including cobalt-based compounds (blue glass), copper alloys (green glass), white calcium antimonate, and yellow lead antimonate. The multianalytical approach of this research has allowed for the distinguishing of the extreme depletion of sodium on the surface of the melted glass fragments due to the exposure to high temperatures during the cremation process, followed by surface weathering in a burial environment. Based on the chemical composition of the bulk of the samples, a model of high temperature viscosity of glass was applied in order to assess the cremation temperature in the pyre, providing relevant information about funerary rituals and cremation technology in Roman Britain.
\end{abstract}

Keywords: Roman glass; cremation; sodium depletion; cremation temperature; XRF; SEM-EDS

\section{Introduction}

\subsection{Cremation in Roman Britain}

Cremation was the main funerary rite in the Early Romano-British period (AD 43-150), and it was gradually replaced by inhumation burial in the mid-second century and throughout the Late Romano-British period (AD 250-410). In the Roman empire, there was a great variation, both geographically and chronologically, in funerary practices. For instance, in Britain cremation was retained long after it had been abandoned and substituted with inhumation on the continent. In fact, especially in the cemeteries in the Northern frontier close to Hadrian's Wall, cremation was the predominant practice [1]. According to archaeological evidence, the dead were placed on a pyre, then burned bones were collected in different types of containers, including glass and ceramic vessels, wooden boxes and fabric or leather bags; ceramic containers represent the most common type. In bustum-type burials, the ashes, bones and debris from the cremation were collected in the pit below the pyre.

The Roman military cemetery of the fort at Birdoswald, discovered in the 1950s, is a representative example. The cemetery is located on the edge of a river cliff, and it was excavated by the Archaeological Projects Department of Historic England in partnership with the Department of Archaeology of the University of Newcastle upon-Tyne in 2009 because it was under threat due to erosion along the nearby river. A peculiarity of the cemetery located in the vicinity of Hadrian's wall is the diversity in the funerary practices within 
single cemeteries, especially regarding the composition of the pits, typology of urns, and organisation of the burials [2]. Several cremation deposits were recovered, most of them contained only charcoal and burnt bones; some pits were cladded with stones and the cremated remains were probably collected in a nailed wooded box; and some urns collecting bones and metal artefacts were also present. The vessels date to the mid-late 2 nd century [3]. These burials are considered "commemorative deposits", as they enclose only a representative sample of material from the pyre, rather than the full cremated remains. This highlights the importance of the cremation event, rather than the deposition in the funerary ritual [3].

In some of the deposits, fragments of glass and glass beads were discovered, together with iron objects. The larger fragments consist of misshapen blue-green glass from vessels, and most of them are distorted by heat, due to the exposure to high temperatures during cremation. In addition, small blue glass chips and blue and green glass beads were found. In Roman cemeteries, glass vessels were common "pyre goods", which probably contained oils and ointments to pour on to the bodies or on the pyre during the ritual [4]. Glass beads in strings and bronze brooches are often found fused adhering to cremated bones, as they were probably placed over the body before the cremation [5].

\subsection{Roman Glassmaking}

A major proportion of ancient glass was made with silica (the former), soda or potash (the flux), and calcium oxide (the stabiliser). In Roman glass, the silica was in the form of sand, while calcium oxide was introduced as shells in the sand [6,7]. The soda was added to reduce the melting temperature of the glass, and for most Roman glass it was obtained from minerals (natron, one of the main sources of this mineral is in Wadi ElNatrun, in Egypt), although in certain regions the ashes of saline plants (salicornia) were used [6,8]. The composition of Roman natron glass artefacts is consistent over time and in different locations, as it was produced in large installations and then exported as raw glass throughout the provinces [9-14].

Transparent pale blue-green is the most common colour of vessels in the 1st-3rd centuries, continuing into the 4th century, and it was used for bottles, household containers, and higher quality tablewares [15]. The blue-green hue was not created by intentionally added colourants, but as a result of the presence of impurities of iron oxide from the sand used to produce the glass [15]. The glass colour could be altered by adding manganese $(\mathrm{Mn})$ or antimony $(\mathrm{Sb})$ minerals. Antimony $(\mathrm{Sb})$ natron glass is often completely colourless whereas the manganese $(\mathrm{Mn})$ natron glass is often blue-green. During recycling antimony and manganese glass became mixed $(\mathrm{Sb}-\mathrm{Mn})$, and this recycled glass also tends to be blue-green [16-18].

\subsection{Research Aims}

The initial aim of this study is to characterise the chemical composition of the glass, and therefore understand the raw materials, colorants and opacifiers used for their production. This investigation was carried out by X-ray fluorescence (XRF) spectroscopy of the surface of the glass fragments from the cremation deposits. Extreme sodium depletion was detected from the surface of the melted glass fragments relative to the unmelted ones, and this was further studied with elemental mapping and morphological investigations.

Secondly, starting from the unaltered glass composition measured by scanning electron microscopy combined with an energy dispersive spectrometer (SEM-EDS) on samples of fresh glass (not the surface) and using mathematical modelling of high temperature viscosity [8], the melting, stiffening, and gathering temperatures of the glass were calculated. In particular, the stiffening and gathering temperatures will give an indication of the temperatures reached during the cremation and the funerary practices. 


\section{Materials and Methods}

The glass samples (Figure 1) were washed with cold water and a soft brush, in order to remove residues of soil. The objects were divided into three main groups: beads and bead fragments (B), fragments of unmelted glass (UG), and melted fragments of glass (MG).

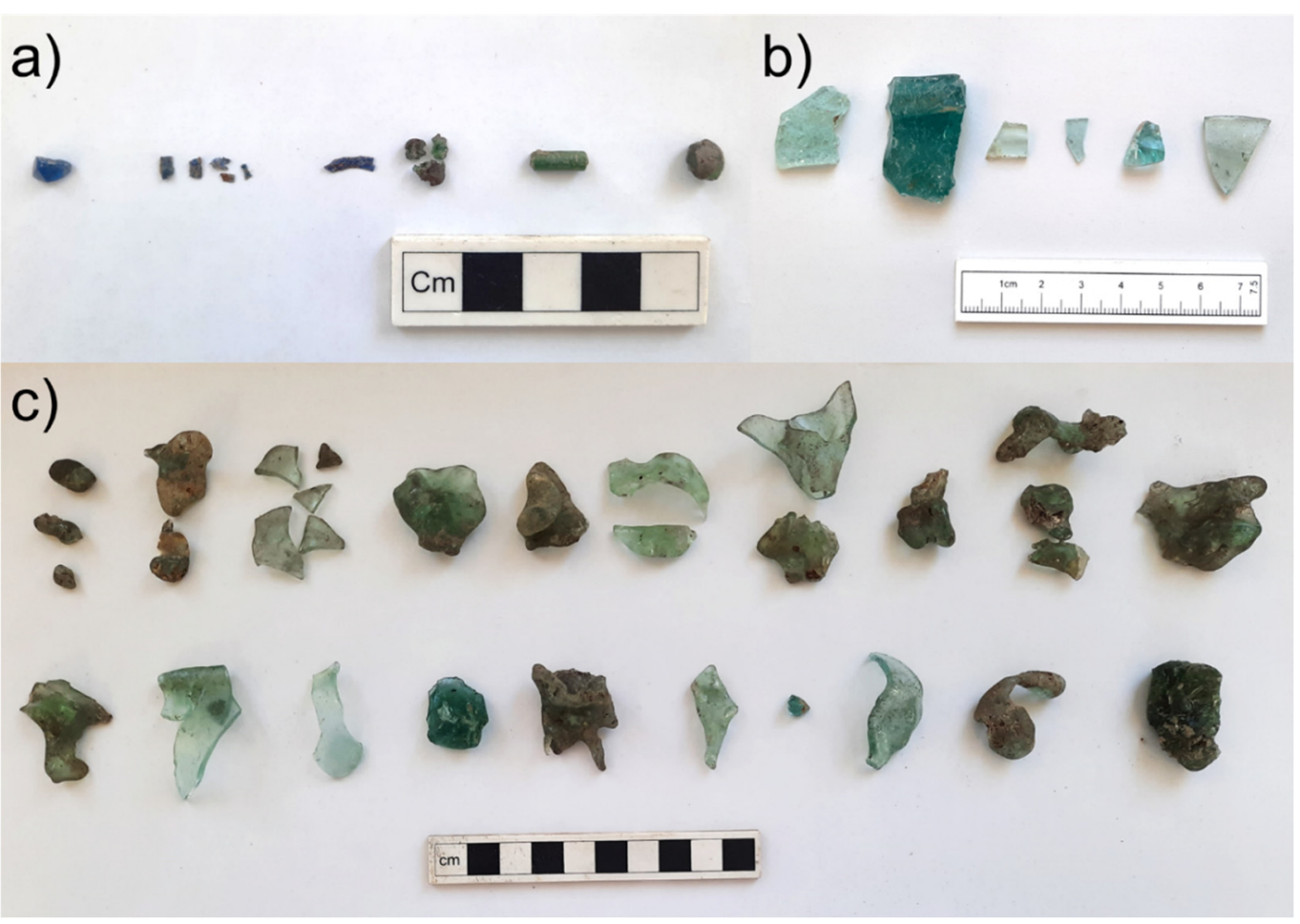

Figure 1. Glass fragments from the assemblage: (a) beads and bead fragments; (b) fragments of unmelted glass; (c) and melted fragments of glass.

Next, the surfaces of the objects were analysed by micro-XRF, using a Bruker M4 Tornado $\mu$-XRF spectrometer. The data were collected at $50 \mathrm{kV}$ and $200 \mu \mathrm{A}$ from an area of $30 \mu$ $\mathrm{m}$ diameter with a vacuum. The tabulated results are averages of at least three analyses and normalised.

Some small samples were collected from the unmelted (UG2, UG3, and UG4) and melted (MG7, MG15, and MG19) glass fragments and were embedded in epoxy resin, ground and polished to a 0.25 -micron finish. The cross-sections were analysed by SEM, using a FEI-Inspect F combined with an energy dispersive spectrometer (EDS) Portsmouth, UK, INCA X-Act. The samples were coated with $15 \mathrm{~nm}$ of carbon and the images were collected using a back-scattered electron (BSE) detector. In this way, unaltered areas in the bulk of the glass could be selected for analysis by EDS, giving the original glass composition. The EDS data were collected at $25 \mathrm{KeV}$ and quantified using Oxford Instruments INCA software. The tabulated results are averages of at least three analyses and normalised. The accuracy and precision of the results were checked by analysing Corning glass standards (Table 1) and was found to be very good, with the exception of sodium oxide. Since the study is focused on the detection of sodium oxide, a calibration curve was plotted with the data collected from the standards and known values, and the results were corrected by using the equation of the calibration curve. The aim of the analyses was to compare the chemical composition of the bulk and the surface of the glass fragments, and to investigate the depletion of sodium oxide on the surface. By necessity, small areas were analysed to determine the surface layer composition (because the layers are thin), and this can itself cause some sodium depletion due to the electron beam, however the EDS data provide corroboration for the low sodium levels detected at the surface of some of the glass by the XRF. 
Table 1. SEM-EDS analysis of glass standards ( $w \mathrm{t} \%)$, average of three analyses, compared to known values (bd = below detection).

\begin{tabular}{|c|c|c|c|c|c|c|c|c|c|c|c|c|c|c|c|c|c|c|c|}
\hline Sam & & & $\mathrm{Na}_{2} \mathrm{O}$ & $\mathrm{MgO}$ & $\mathrm{Al}_{2} \mathrm{O}_{3}$ & $\mathrm{SiO}_{2}$ & $\mathrm{P}_{2} \mathrm{O}_{5}$ & $\mathrm{SO}_{3}$ & $\mathrm{~K}_{2} \mathrm{O}$ & $\mathrm{CaO}$ & $\mathrm{TiO}_{2}$ & $\mathrm{MnO}$ & $\mathrm{Fe}_{2} \mathrm{O}_{3}$ & $\mathrm{CoO}$ & $\mathrm{CuO}$ & $\mathrm{ZnO}$ & $\mathrm{Sb}_{2} \mathrm{O}_{5}$ & $\mathrm{BaO}$ & $\overline{\mathrm{PbO}}$ \\
\hline \multirow{3}{*}{ Corning A } & Measured & & 13.15 & 2.73 & 0.98 & 67.64 & $\mathrm{bd}$ & 0.27 & 3.16 & 5.25 & 0.77 & 1.03 & 1.04 & 0.20 & 1.28 & 0.07 & 1.69 & 0.59 & 0.13 \\
\hline & & & 0.26 & 0.13 & 0.21 & 0.46 & $\mathrm{bd}$ & 0.13 & 0.07 & 0.12 & 0.20 & 0.08 & 0.12 & 0.02 & 0.08 & 0.05 & 0.33 & 0.15 & 0.22 \\
\hline & Known & & 14.30 & 2.66 & 1.00 & 66.76 & 0.13 & 0.10 & 2.87 & 5.03 & 0.79 & 1.00 & 1.09 & 0.17 & 1.17 & 0.04 & 1.75 & 0.56 & 0.12 \\
\hline \multirow{3}{*}{ Corning B } & Measured & Avg & 15.86 & 1.09 & 4.32 & 62.15 & 0.85 & 0.55 & 1.15 & 8.90 & 0.13 & 0.30 & 0.29 & 0.07 & 3.07 & 0.24 & 0.43 & $\mathrm{bd}$ & 0.55 \\
\hline & & & 0.18 & 0.08 & 0.04 & 0.70 & 0.25 & 0.14 & 0.08 & 0.16 & 0.03 & 0.12 & 0.10 & 0.06 & 0.06 & 0.18 & 0.38 & 0.08 & 0.12 \\
\hline & Known & & 17.00 & 1.03 & 4.36 & 61.52 & 0.82 & 0.50 & 1.00 & 8.56 & 0.09 & 0.25 & 0.34 & 0.05 & 2.66 & 0.19 & 0.46 & 0.12 & 0.61 \\
\hline \multirow{3}{*}{ Corning C } & Measured & Avg & 1.18 & 2.72 & 0.80 & 34.65 & $\mathrm{bd}$ & $\mathrm{bd}$ & 3.01 & 5.20 & 0.87 & 0.07 & 0.35 & 0.18 & 1.23 & 0.09 & 0.39 & 12.56 & 36.7 \\
\hline & & & 0.18 & 0.03 & 0.22 & 0.29 & $\mathrm{bd}$ & $\mathrm{bd}$ & 0.07 & 0.14 & 0.07 & 0.06 & 0.05 & 0.08 & 0.06 & 0.14 & 0.51 & 0.15 & 0.32 \\
\hline & Known & & 1.07 & 2.76 & 0.87 & 35.88 & 0.14 & 0.10 & 2.84 & 5.07 & 0.79 & $\mathrm{bd}$ & 0.34 & 0.18 & 1.13 & 0.05 & 0.03 & 11.40 & 36.7 \\
\hline \multirow{3}{*}{ Corning D } & Measured & Avg & 1.36 & 4.18 & 5.04 & 54.80 & 3.85 & 0.42 & 11.95 & 15.08 & 0.45 & 0.44 & 0.48 & $\mathrm{bd}$ & 0.34 & 0.14 & 0.80 & 0.41 & 0.27 \\
\hline & & & 0.18 & 0.23 & 0.03 & 0.34 & 0.17 & 0.16 & 0.11 & 0.12 & 0.10 & 0.08 & 0.14 & $\mathrm{bd}$ & 0.20 & 0.12 & 0.24 & 0.21 & 0.11 \\
\hline & Known & & 1.20 & 3.94 & 5.30 & 54.65 & 3.93 & 0.30 & 11.30 & 14.80 & 0.38 & 0.55 & 0.52 & 0.02 & 0.38 & 0.10 & 0.97 & 0.51 & 0.48 \\
\hline
\end{tabular}

To evaluate the melting, stiffening, and gathering temperatures of the glass, mathematical modelling based on the composition and high temperature viscosity of glass was used $[19,20]$. This model was developed by introducing the influence of the composition on viscosity into the Vogel-Fulcher-Tammann equation, which describes the glass viscosity as a function of the temperature for a Newtonian fluid:

$$
\log (\eta / d P a s)=A+B /(T-T 0)
$$

where $\eta$ is the viscosity, $\mathrm{A}, \mathrm{B}$ and $\mathrm{T}_{0}$ are composition-dependent constants, which are calculated using contributions from each component oxide, and $\mathrm{T}$ is the temperature. Lakatos' values of $\mathrm{A}, \mathrm{B}$ and $\mathrm{T}_{0}$ constants were used to calculate the temperatures that are necessary to melt $(\log (\eta / \mathrm{dPa} \mathrm{s})=2.9)$, gather $(\log (\eta / \mathrm{dPa} s)=3.3)$, and stiffen $(\log (\eta / \mathrm{dPa} s)=8)$ the glass $[19,20]$. The viscosities calculated by this model have a good accuracy (standard deviation $\sim 3.0^{\circ} \mathrm{C}$ ) if the compositions are calculated on a molecular basis. The Lakatos model was selected, as it has been validated in the study of Roman blue-green glass samples with composition similar to the glass in this study [21]. This model was applied by using the chemical composition acquired by SEM-EDS of the bulk of the cross-sections of the fragments (UG2, UG3, UG4, MG7, MG15, and MG19) because it was not affected by chemical and morphological changes due to cremation.

\section{Results}

A summary of the results is given in Table 2 and the full analytical results are given in Tables 3-6. The objects are divided into beads and bead fragments (B), fragments of unmelted glass (UG) and melted fragments of glass (MG). The probable glass type is also given in the final column.

\subsection{Chemical Characteristics of the Different Types of Glass}

Examining the ratios of elements in the glass can reveal more information about each of the glass categories, differentiating raw materials and colourants used in their production.

In Figure 2a, by plotting the XRF results for $\mathrm{Na}_{2} \mathrm{O}$ and the sum of $\mathrm{MgO}, \mathrm{CaO}, \mathrm{K}_{2} \mathrm{O}$, and $\mathrm{P}_{2} \mathrm{O}_{5}$ contents of the surface of the glass, the data provide information about the alkali source used in the mix (in the graphs triangle symbols indicate beads and the colour corresponds to the colour of the fragments; square and round symbols correspond to unmelted and melted blue-green glass fragments). The fragments are characterised by high variability in the sodium (discussed later) but consistently low concentrations of $\mathrm{MgO}, \mathrm{CaO}$, $\mathrm{K}_{2} \mathrm{O}$, and $\mathrm{P}_{2} \mathrm{O}_{5}$, indicating that the alkali source used in the formulation is natron instead of plant ashes, as expected. The division into "low magnesia" and "high magnesia" glasses identifies those made using natron and plant ashes, respectively [8]. Indeed, plant-based glasses have high potash and magnesia and phosphorous contents, while natron-based 
glasses show low contents of these compounds [8]. Furthermore, the phosphorous content has been used in the discrimination of these types of glass [22].
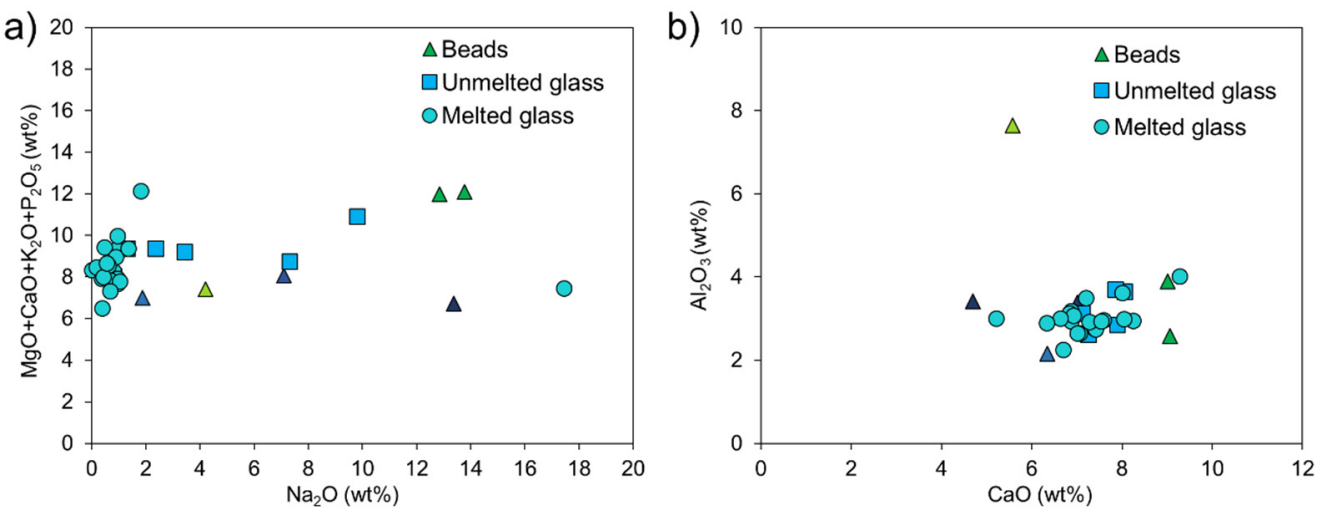

Figure 2. Surface $X R F$ data for $\mathrm{Na}_{2} \mathrm{O}$ and the sum of $\mathrm{MgO}, \mathrm{CaO}, \mathrm{K}_{2} \mathrm{O}$, and $\mathrm{P}_{2} \mathrm{O}_{5}(\mathbf{a})$, and $\mathrm{CaO}$ and $\mathrm{Al}_{2} \mathrm{O}_{3}$ (b) in the glass fragments; note the low $\mathrm{Na}_{2} \mathrm{O}$ concentrations in the melted glass discussed later.

Table 2. Summary of the glass fragments from Birdoswald cemetery.

\begin{tabular}{ccc}
\hline Sample ID & Description & Type of Glass \\
\hline B1 & Transparent blue bead & Sb glass \\
B2 & Opaque dark blue fragment & Sb-Mn glass \\
B3 & Opaque dark blue fragment & Sb-Mn glass \\
B4 & Green bead (burned) & Sb-Mn glass \\
B5 & Opaque light green bead & Sb-Mn glass \\
B6 & Green bead (burned) & Sb-Mn glass \\
UG1 & Blue-green fragment & Low-Mn glass \\
UG2 & Blue-green fragment & Sb-Mn glass \\
UG3 & Blue-green fragment & Sb-Mn glass \\
UG4 & Blue-green fragment & Sb-Mn glass \\
UG5 & Blue-green fragment & Sb-Mn glass \\
UG6 & Blue-green fragment & Sb-Mn glass \\
MG1 & Melted blue-green fragment & Sb-Mn glass \\
MG2 & Melted blue-green fragment & Sb-Mn glass \\
MG3 & Melted blue-green fragment & Sb-Mn glass \\
MG4 & Melted blue-green fragment & Sb-Mn glass \\
MG5 & Melted blue-green fragment & Sb-Mn glass \\
MG6 & Melted blue-green fragment & Sb-Mn glass \\
MG7 & Melted blue-green fragment & Sb-Mn glass \\
MG8 & Melted blue-green fragment & Sb-Mn glass \\
MG9 & Melted blue-green fragment & Sb-Mn glass \\
MG10 & Melted blue-green fragment & Sb-Mn glass \\
MG11 & Melted blue-green fragment & Sb-Mn glass \\
MG12 & Melted blue-green fragment & Sb-Mn glass \\
MG13 & Melted blue-green fragment & Sb-Mn glass \\
MG14 & Melted blue-green fragment & Sb-Mn glass \\
MG15 & Melted blue-green fragment & Sb-Mn glass \\
MG16 & Melted blue-green fragment & Sb-Mn glass \\
MG17 & Melted blue-green fragment & Sb-Mn glass \\
MG18 & Sb-Mn glass \\
MG19 & Melted blue-green fragment & Sb-Mn glass \\
MG20 & Melted blue-green fragment & Sb-Mn glass \\
\hline & & \\
& & Slue-green fragment \\
\hline
\end{tabular}


Table 3. Chemical composition (microXRF, wt \% oxides, normalised) of the surface of glass beads and fragments (bd = below detection).

\begin{tabular}{|c|c|c|c|c|c|c|c|c|c|c|c|c|c|c|c|c|c|c|c|}
\hline \multicolumn{2}{|c|}{ Sample } & \multirow{3}{*}{$\begin{array}{c}\mathrm{Na}_{2} \mathbf{O} \\
1.89 \\
0.79\end{array}$} & \multirow{3}{*}{$\begin{array}{c}\mathbf{M g O} \\
0.27 \\
0.03\end{array}$} & \multirow{3}{*}{$\begin{array}{c}\mathbf{A l}_{\mathbf{2}} \mathbf{O}_{3} \\
2.16 \\
0.18\end{array}$} & \multirow{3}{*}{$\begin{array}{c}\mathrm{SiO}_{2} \\
85.68 \\
0.93\end{array}$} & \multirow{3}{*}{$\begin{array}{l}\mathbf{S O}_{3} \\
0.46 \\
0.05\end{array}$} & \multirow{3}{*}{$\begin{array}{r}\mathbf{K}_{\mathbf{2}} \mathbf{O} \\
0.37 \\
0.18\end{array}$} & \multirow{3}{*}{$\begin{array}{c}\mathrm{CaO} \\
6.35 \\
0.22\end{array}$} & \multirow{3}{*}{$\begin{array}{c}\mathbf{T i O}_{\mathbf{2}} \\
0.07 \\
0.01\end{array}$} & \multirow{3}{*}{$\begin{array}{c}\mathbf{M n O}_{\mathbf{2}} \\
0.06 \\
0.01\end{array}$} & \multirow{3}{*}{$\begin{array}{c}\mathrm{Fe}_{2} \mathbf{O}_{3} \\
0.99 \\
0.11\end{array}$} & \multirow{3}{*}{$\begin{array}{l}\mathrm{CoO} \\
0.08 \\
0.01\end{array}$} & \multirow{3}{*}{$\begin{array}{l}\mathrm{CuO} \\
0.32 \\
0.03\end{array}$} & \multirow{3}{*}{$\begin{array}{l}\text { SrO } \\
0.08 \\
0.01\end{array}$} & \multirow{3}{*}{$\begin{array}{c}\mathbf{S b}_{\mathbf{2}} \mathbf{O}_{\mathbf{5}} \\
0.95 \\
0.11\end{array}$} & \multirow{3}{*}{$\begin{array}{c}\text { PbO } \\
0.25 \\
0.04\end{array}$} & \multirow{3}{*}{$\begin{array}{c}\mathbf{P}_{2} \mathbf{O}_{5} \\
\text { bd } \\
\text { bd }\end{array}$} & \multirow{3}{*}{$\begin{array}{c}\text { ZnO } \\
0.01 \\
0.00\end{array}$} & \multirow{3}{*}{$\begin{array}{c}\mathrm{SnO}_{2} \\
\mathrm{bd} \\
\mathrm{bd}\end{array}$} \\
\hline & Avg & & & & & & & & & & & & & & & & & & \\
\hline B1 & std & & & & & & & & & & & & & & & & & & \\
\hline \multirow{2}{*}{ B2 } & Avg & 13.37 & 0.15 & 3.42 & 66.60 & 0.03 & 1.86 & 4.70 & 0.14 & 0.20 & 1.63 & 0.35 & 0.45 & 0.05 & 4.42 & 2.28 & $\mathrm{bd}$ & 0.31 & 0.03 \\
\hline & std & 2.10 & 0.21 & 1.78 & 1.80 & 0.03 & 0.20 & 0.81 & 0.16 & 0.20 & 1.04 & 0.17 & 0.08 & 0.00 & 2.22 & 1.07 & $\mathrm{bd}$ & 0.05 & 0.06 \\
\hline \multirow[b]{2}{*}{ B3 } & Avg & 7.11 & 0.19 & 3.38 & 73.95 & 0.35 & 0.50 & 7.02 & 0.06 & 0.70 & 2.05 & 0.34 & 0.57 & 0.05 & 1.79 & 1.59 & 0.34 & 0.01 & $\mathrm{bd}$ \\
\hline & std & 0.86 & 0.07 & 1.11 & 5.07 & 0.06 & 0.08 & 0.50 & 0.01 & 0.56 & 2.17 & 0.13 & 0.14 & 0.02 & 0.35 & 0.73 & 0.67 & 0.00 & $\mathrm{bd}$ \\
\hline \multirow{2}{*}{ B4 } & Avg & 13.78 & 1.51 & 3.90 & 66.22 & 0.25 & 1.29 & 9.01 & 0.13 & 0.19 & 1.34 & 0.01 & 1.47 & 0.07 & 0.11 & 0.27 & 0.27 & 0.03 & 0.15 \\
\hline & std & 1.89 & 0.09 & 1.03 & 0.99 & 0.02 & 0.04 & 0.34 & 0.01 & 0.00 & 0.10 & 0.00 & 0.17 & 0.03 & 0.08 & 0.08 & 0.10 & 0.00 & 0.12 \\
\hline B5 & std & 0.70 & 0.07 & 0.40 & 0.76 & $\mathrm{bd}$ & 0.03 & 0.17 & 0.01 & 0.01 & 0.05 & $\mathrm{bd}$ & 0.13 & 0.01 & 0.09 & 0.56 & $\mathrm{bd}$ & 0.00 & 0.35 \\
\hline \multirow{2}{*}{ B6 } & Avg & 12.85 & 1.51 & 2.58 & 68.27 & 0.26 & 1.29 & 9.06 & 0.12 & 0.19 & 1.30 & 0.01 & 1.54 & 0.08 & 0.18 & 0.31 & 0.12 & 0.03 & 0.29 \\
\hline & std & 1.36 & 0.06 & 0.12 & 0.94 & 0.01 & 0.10 & 0.28 & 0.01 & 0.01 & 0.04 & 0.00 & 0.10 & 0.01 & 0.04 & 0.02 & 0.02 & 0.00 & 0.06 \\
\hline
\end{tabular}

Table 4. Chemical composition (microXRF, wt $\%$ oxides, normalised) of the surface of unmelted glass fragments (bd = below detection).

\begin{tabular}{|c|c|c|c|c|c|c|c|c|c|c|c|c|c|c|c|c|c|c|c|}
\hline \multicolumn{2}{|c|}{ Sample } & \multirow{3}{*}{$\begin{array}{c}\mathrm{Na}_{\mathbf{2}} \mathbf{O} \\
9.83 \\
0.73\end{array}$} & \multirow{3}{*}{$\begin{array}{c}\text { MgO } \\
0.19 \\
0.02\end{array}$} & \multirow{3}{*}{$\begin{array}{c}\mathrm{Al}_{\mathbf{2}} \mathbf{O}_{3} \\
2.61 \\
0.06\end{array}$} & \multirow{3}{*}{$\begin{array}{c}\mathrm{SiO}_{2} \\
75.86 \\
0.23 \\
\end{array}$} & \multirow{3}{*}{$\begin{array}{l}\mathrm{SO}_{3} \\
0.09 \\
0.01\end{array}$} & \multirow{3}{*}{$\begin{array}{c}\mathbf{K}_{\mathbf{2}} \mathbf{O} \\
3.42 \\
0.92 \\
\end{array}$} & \multirow{3}{*}{$\begin{array}{l}\mathrm{CaO} \\
7.27 \\
0.08\end{array}$} & \multirow{3}{*}{$\begin{array}{c}\mathrm{TiO}_{\mathbf{2}} \\
0.05 \\
0.00 \\
\end{array}$} & \multirow{3}{*}{$\begin{array}{c}\mathbf{M n O}_{\mathbf{2}} \\
0.29 \\
0.01 \\
\end{array}$} & \multirow{3}{*}{$\begin{array}{c}\mathbf{F e}_{\mathbf{2}} \mathbf{O}_{3} \\
0.31 \\
0.01\end{array}$} & \multirow{3}{*}{$\begin{array}{c}\mathrm{CoO} \\
\mathrm{bd} \\
\mathrm{bd}\end{array}$} & \multirow{3}{*}{$\begin{array}{c}\mathrm{CuO} \\
\mathrm{bd} \\
\mathrm{bd}\end{array}$} & \multirow{3}{*}{$\begin{array}{l}\text { SrO } \\
0.05 \\
0.00\end{array}$} & \multirow{3}{*}{$\begin{array}{c}\mathrm{Sb}_{2} \mathrm{O}_{5} \\
\mathrm{bd} \\
\mathrm{bd}\end{array}$} & \multirow{3}{*}{$\begin{array}{c}\mathrm{PbO} \\
\mathrm{bd} \\
\mathrm{bd}\end{array}$} & \multirow{3}{*}{$\begin{array}{c}\mathbf{P}_{2} \mathbf{O}_{5} \\
b d \\
b d\end{array}$} & \multirow{3}{*}{$\begin{array}{c}\mathrm{ZnO} \\
\mathrm{bd} \\
\mathrm{bd}\end{array}$} & \multirow{3}{*}{$\begin{array}{c}\mathrm{SnO}_{2} \\
\mathrm{bd} \\
\mathrm{bd}\end{array}$} \\
\hline & Avg & & & & & & & & & & & & & & & & & & \\
\hline UG1 & std & & & & & & & & & & & & & & & & & & \\
\hline \multirow{2}{*}{ UG2 } & Avg & 2.39 & 0.41 & 3.64 & 82.25 & 0.47 & 0.86 & 8.07 & 0.10 & 0.32 & 1.01 & bd & 0.01 & 0.05 & 0.35 & 0.04 & $\mathrm{bd}$ & $\mathrm{bd}$ & $\mathrm{bd}$ \\
\hline & std & 0.52 & 0.05 & 0.66 & 0.53 & 0.11 & 0.03 & 0.11 & 0.00 & 0.01 & 0.12 & $\mathrm{bd}$ & 0.00 & 0.00 & 0.01 & 0.00 & $\mathrm{bd}$ & $\mathrm{bd}$ & $\mathrm{bd}$ \\
\hline \multirow[b]{2}{*}{ UG3 } & Avg & 7.32 & 0.25 & 2.87 & 78.87 & 0.42 & 1.07 & 7.40 & 0.09 & 0.63 & 0.67 & $\mathrm{bd}$ & 0.01 & 0.05 & 0.25 & 0.06 & $\mathrm{bd}$ & 0.01 & $\mathrm{bd}$ \\
\hline & std & 0.11 & 0.05 & 0.02 & 0.12 & 0.00 & 0.03 & 0.15 & 0.01 & 0.01 & 0.01 & $\mathrm{bd}$ & 0.00 & 0.00 & 0.02 & 0.00 & $\mathrm{bd}$ & 0.00 & bd \\
\hline UG4 & Avg & 3.45 & 0.34 & 2.85 & 82.25 & 0.36 & 0.95 & 7.90 & 0.09 & 0.42 & 0.82 & $\mathrm{bd}$ & 0.01 & 0.05 & 0.18 & 0.29 & $\mathrm{bd}$ & 0.01 & bd \\
\hline \multirow[b]{2}{*}{ UG5 } & Avg & 0.85 & 0.28 & 3.11 & 85.61 & 0.48 & 0.60 & 7.14 & 0.08 & 0.44 & 0.76 & $\mathrm{bd}$ & 0.01 & 0.05 & 0.49 & 0.08 & $\mathrm{bd}$ & $\mathrm{bd}$ & bd \\
\hline & std & 0.15 & 0.03 & 0.35 & 0.22 & 0.02 & 0.00 & 0.19 & 0.00 & 0.01 & 0.01 & $\mathrm{bd}$ & 0.00 & 0.00 & 0.02 & 0.00 & $\mathrm{bd}$ & $\mathrm{bd}$ & $\mathrm{bd}$ \\
\hline \multirow{2}{*}{ UG6 } & Avg & 1.34 & 0.52 & 3.69 & 83.60 & 0.51 & 0.96 & 7.87 & 0.10 & 0.32 & 0.65 & bd & 0.01 & 0.06 & 0.32 & 0.04 & bd & 0.01 & $\mathrm{bd}$ \\
\hline & std & 0.32 & 0.22 & 0.39 & 1.20 & 0.03 & 0.06 & 0.22 & 0.01 & 0.01 & 0.07 & bd & 0.00 & 0.00 & 0.01 & 0.00 & $\mathrm{bd}$ & 0.00 & bd \\
\hline
\end{tabular}


Table 5. Chemical composition (microXRF, wt $\%$ oxides, normalised) of the surface of melted glass fragments (bd $=$ below detection).

\begin{tabular}{|c|c|c|c|c|c|c|c|c|c|c|c|c|c|c|c|c|c|c|c|}
\hline \multicolumn{2}{|c|}{ Sample } & \multirow{2}{*}{$\begin{array}{c}\mathrm{Na}_{2} \mathrm{O} \\
0.83 \\
0.11\end{array}$} & \multirow{2}{*}{$\begin{array}{c}\text { MgO } \\
0.32 \\
0.07\end{array}$} & \multirow{2}{*}{$\begin{array}{c}\mathrm{Al}_{2} \mathrm{O}_{3} \\
\begin{array}{c}2.76 \\
0.03\end{array}\end{array}$} & \multirow{2}{*}{$\begin{array}{c}\mathrm{SiO}_{2} \\
86.46 \\
0.17\end{array}$} & \multirow{2}{*}{$\begin{array}{l}\mathrm{SO}_{3} \\
0.32 \\
0.02\end{array}$} & \multirow{2}{*}{$\begin{array}{c}\mathrm{K}_{2} \mathrm{O} \\
0.52 \\
0.00\end{array}$} & \multirow{2}{*}{$\begin{array}{c}\text { CaO } \\
7.41 \\
0.05\end{array}$} & \multirow{2}{*}{$\begin{array}{c}\mathrm{TiO}_{2} \\
0.08 \\
0.00\end{array}$} & \multirow{2}{*}{$\begin{array}{c}\mathrm{MnO}_{2} \\
\begin{array}{l}0.31 \\
0.01\end{array}\end{array}$} & \multirow{2}{*}{$\begin{array}{c}\mathrm{Fe}_{2} \mathrm{O}_{3} \\
\begin{array}{l}0.54 \\
0.01\end{array}\end{array}$} & \multirow{2}{*}{$\begin{array}{c}\mathrm{CoO} \\
\begin{array}{l}\mathrm{bd} \\
\mathrm{bd}\end{array}\end{array}$} & \multirow{2}{*}{$\begin{array}{c}\text { CuO } \\
0.01 \\
0.00\end{array}$} & \multirow{2}{*}{$\begin{array}{l}\text { SrO } \\
0.05 \\
0.00\end{array}$} & \multirow{2}{*}{$\begin{array}{c}\mathbf{S b}_{\mathbf{2}} \mathbf{O}_{\mathbf{5}} \\
\begin{array}{c}0.32 \\
0.01\end{array}\end{array}$} & \multirow{2}{*}{$\begin{array}{c}\mathrm{PbO} \\
0.04 \\
0.00\end{array}$} & \multirow{2}{*}{$\begin{array}{c}\mathrm{P}_{2} \mathrm{O}_{5} \\
\begin{array}{c}\mathrm{bd} \\
\mathrm{bd}\end{array}\end{array}$} & \multirow{2}{*}{$\begin{array}{r}\mathrm{ZnO} \\
\begin{array}{l}\mathrm{bd} \\
\mathrm{bd}\end{array}\end{array}$} & \multirow{2}{*}{$\begin{array}{r}\mathrm{SnO}_{2} \\
\mathrm{bd} \\
\mathrm{bd}\end{array}$} \\
\hline MG1 & $\begin{array}{l}\text { Avg } \\
\text { std }\end{array}$ & & & & & & & & & & & & & & & & & & \\
\hline MG2 & $\begin{array}{l}\text { Avg } \\
\text { std }\end{array}$ & $\begin{array}{l}0.94 \\
0.36\end{array}$ & $\begin{array}{l}0.41 \\
0.07\end{array}$ & $\begin{array}{l}2.94 \\
0.13\end{array}$ & $\begin{array}{c}85.10 \\
0.38\end{array}$ & $\begin{array}{l}0.32 \\
0.01\end{array}$ & \begin{tabular}{l|}
0.63 \\
0.02
\end{tabular} & $\begin{array}{l}8.26 \\
0.08\end{array}$ & \begin{tabular}{|l|}
0.09 \\
0.00
\end{tabular} & $\begin{array}{l}0.35 \\
0.01\end{array}$ & $\begin{array}{l}0.58 \\
0.02\end{array}$ & $\begin{array}{l}\mathrm{bd} \\
\mathrm{bd}\end{array}$ & $\begin{array}{l}0.01 \\
0.00\end{array}$ & $\begin{array}{l}0.05 \\
0.00\end{array}$ & $\begin{array}{l}0.26 \\
0.03\end{array}$ & $\begin{array}{l}0.04 \\
0.00\end{array}$ & $\begin{array}{l}\mathrm{bd} \\
\mathrm{bd}\end{array}$ & $\begin{array}{l}\mathrm{bd} \\
\mathrm{bd}\end{array}$ & $\begin{array}{l}\mathrm{bd} \\
\mathrm{bd}\end{array}$ \\
\hline MG3 & $\begin{array}{l}\text { Avg } \\
\text { std }\end{array}$ & $\begin{array}{l}0.97 \\
0.07 \\
\end{array}$ & $\begin{array}{l}0.33 \\
0.02 \\
\end{array}$ & $\begin{array}{l}2.93 \\
0.18 \\
\end{array}$ & $\begin{array}{c}86.60 \\
0.48 \\
\end{array}$ & $\begin{array}{l}0.31 \\
0.01 \\
\end{array}$ & $\begin{array}{l}0.45 \\
0.04 \\
\end{array}$ & $\begin{array}{l}6.89 \\
0.23 \\
\end{array}$ & $\begin{array}{l}0.08 \\
0.00 \\
\end{array}$ & $\begin{array}{l}0.29 \\
0.01 \\
\end{array}$ & $\begin{array}{l}0.55 \\
0.03 \\
\end{array}$ & $\begin{array}{l}\mathrm{bd} \\
\mathrm{bd} \\
\end{array}$ & $\begin{array}{l}0.01 \\
0.00 \\
\end{array}$ & $\begin{array}{l}0.05 \\
0.01 \\
\end{array}$ & $\begin{array}{l}0.48 \\
0.04 \\
\end{array}$ & $\begin{array}{l}0.03 \\
0.00 \\
\end{array}$ & $\begin{array}{l}\mathrm{bd} \\
\mathrm{bd}\end{array}$ & $\begin{array}{l}\mathrm{bd} \\
\mathrm{bd}\end{array}$ & $\begin{array}{l}\mathrm{bd} \\
\mathrm{bd}\end{array}$ \\
\hline MG4 & $\begin{array}{l}\text { Avg } \\
\text { stdd }\end{array}$ & $\begin{array}{l}0.98 \\
0.28\end{array}$ & $\begin{array}{l}0.36 \\
0.03\end{array}$ & $\begin{array}{l}3.17 \\
0.34\end{array}$ & $\begin{array}{l}86.34 \\
0.24\end{array}$ & $\begin{array}{l}0.35 \\
0.01\end{array}$ & $\begin{array}{l}0.66 \\
0.05\end{array}$ & $\begin{array}{l}6.88 \\
0.08\end{array}$ & $\begin{array}{l}0.09 \\
0.01\end{array}$ & $\begin{array}{l}0.32 \\
0.01\end{array}$ & $\begin{array}{l}0.60 \\
0.05\end{array}$ & $\begin{array}{l}\mathrm{bd} \\
\mathrm{bd}\end{array}$ & $\begin{array}{l}\text { bd } \\
\text { bd }\end{array}$ & $\begin{array}{l}0.05 \\
0.00\end{array}$ & $\begin{array}{l}0.14 \\
0.04\end{array}$ & $\begin{array}{l}0.04 \\
0.00\end{array}$ & $\begin{array}{l}\text { bd } \\
\text { bd }\end{array}$ & $\begin{array}{l}0.01 \\
0.00\end{array}$ & $\begin{array}{l}\mathrm{bd} \\
\mathrm{bd}\end{array}$ \\
\hline MG5 & $\begin{array}{l}\text { Avg } \\
\text { stdd }\end{array}$ & $\begin{array}{l}0.71 \\
0.24\end{array}$ & $\begin{array}{l}0.27 \\
0.02\end{array}$ & $\begin{array}{l}2.64 \\
0.09\end{array}$ & $\begin{array}{l}87.12 \\
0.43\end{array}$ & $\begin{array}{l}0.30 \\
0.01\end{array}$ & $\begin{array}{l}0.49 \\
0.07\end{array}$ & $\begin{array}{l}7.07 \\
0.17\end{array}$ & $\begin{array}{l}0.08 \\
0.00\end{array}$ & $\begin{array}{l}0.29 \\
0.01\end{array}$ & $\begin{array}{l}0.51 \\
0.02\end{array}$ & $\begin{array}{l}\text { bd } \\
\text { bd }\end{array}$ & $\begin{array}{l}0.01 \\
0.00\end{array}$ & $\begin{array}{l}0.05 \\
0.00\end{array}$ & $\begin{array}{l}0.41 \\
0.05 \\
\end{array}$ & $\begin{array}{l}0.03 \\
0.00\end{array}$ & $\begin{array}{l}\text { bd } \\
\text { bd }\end{array}$ & $\begin{array}{l}\text { bd } \\
\text { bd }\end{array}$ & $\begin{array}{l}\mathrm{bd} \\
\mathrm{bd}\end{array}$ \\
\hline MG6 & $\begin{array}{l}\text { Avg } \\
\text { std }\end{array}$ & $\begin{array}{l}0.04 \\
0.07\end{array}$ & $\begin{array}{l}0.38 \\
0.05 \\
\end{array}$ & $\begin{array}{l}2.74 \\
0.08 \\
\end{array}$ & $\begin{array}{c}87.23 \\
0.17\end{array}$ & $\begin{array}{l}0.31 \\
0.01 \\
\end{array}$ & $\begin{array}{l}0.49 \\
0.02 \\
\end{array}$ & $\begin{array}{l}7.43 \\
0.03 \\
\end{array}$ & $\begin{array}{l}0.08 \\
0.00\end{array}$ & $\begin{array}{l}0.30 \\
0.00 \\
\end{array}$ & $\begin{array}{l}0.54 \\
0.01 \\
\end{array}$ & $\begin{array}{l}\mathrm{bd} \\
\mathrm{bd}\end{array}$ & $\begin{array}{l}0.01 \\
0.00 \\
\end{array}$ & $\begin{array}{l}0.05 \\
0.00 \\
\end{array}$ & $\begin{array}{l}0.36 \\
0.03 \\
\end{array}$ & $\begin{array}{l}0.04 \\
0.00 \\
\end{array}$ & $\begin{array}{l}\mathrm{bd} \\
\mathrm{bd}\end{array}$ & $\begin{array}{l}\mathrm{bd} \\
\mathrm{bd}\end{array}$ & $\begin{array}{l}\mathrm{bd} \\
\mathrm{bd}\end{array}$ \\
\hline MG7 & $\begin{array}{l}\text { Avg } \\
\text { std }\end{array}$ & $\begin{array}{l}1.83 \\
0.58\end{array}$ & $\begin{array}{l}0.64 \\
0.04\end{array}$ & $\begin{array}{l}4.01 \\
0.32\end{array}$ & $\begin{array}{c}79.72 \\
0.33\end{array}$ & $\begin{array}{l}0.28 \\
0.04\end{array}$ & $\begin{array}{l}2.19 \\
0.13\end{array}$ & $\begin{array}{l}9.29 \\
0.36\end{array}$ & $\begin{array}{l}0.17 \\
0.04\end{array}$ & $\begin{array}{l}0.43 \\
0.03\end{array}$ & $\begin{array}{l}0.94 \\
0.10\end{array}$ & $\begin{array}{l}\text { bd } \\
\text { bd }\end{array}$ & $\begin{array}{l}0.06 \\
0.02\end{array}$ & $\begin{array}{l}0.06 \\
0.01\end{array}$ & $\begin{array}{l}0.29 \\
0.02\end{array}$ & $\begin{array}{l}0.04 \\
0.00\end{array}$ & $\begin{array}{l}\mathrm{bd} \\
\mathrm{bd}\end{array}$ & $\begin{array}{l}0.02 \\
0.01\end{array}$ & $\begin{array}{l}\text { bd } \\
\text { bd }\end{array}$ \\
\hline MG8 & $\begin{array}{l}\text { Avg } \\
\text { stdd }\end{array}$ & $\begin{array}{l}0.49 \\
0.21\end{array}$ & $\begin{array}{l}0.60 \\
0.07\end{array}$ & $\begin{array}{l}2.95 \\
0.03\end{array}$ & $\begin{array}{l}85.38 \\
0.67\end{array}$ & $\begin{array}{l}0.17 \\
0.01\end{array}$ & $\begin{array}{l}1.21 \\
0.11\end{array}$ & $\begin{array}{l}7.61 \\
0.35\end{array}$ & $\begin{array}{l}0.08 \\
0.00\end{array}$ & $\begin{array}{l}0.32 \\
0.01 \\
\end{array}$ & $\begin{array}{l}0.59 \\
0.02\end{array}$ & $\begin{array}{l}\mathrm{bd} \\
\mathrm{bd}\end{array}$ & $\begin{array}{l}0.02 \\
0.00\end{array}$ & $\begin{array}{l}0.06 \\
0.00\end{array}$ & $\begin{array}{l}0.45 \\
0.02\end{array}$ & $\begin{array}{l}0.03 \\
0.00\end{array}$ & $\begin{array}{l}\text { bd } \\
\text { bd }\end{array}$ & $\begin{array}{l}0.01 \\
0.00\end{array}$ & $\begin{array}{l}\mathrm{bd} \\
\mathrm{bd}\end{array}$ \\
\hline MG9 & $\begin{array}{l}\text { Avg } \\
\text { std }\end{array}$ & $\begin{array}{l}0.39 \\
0.18 \\
\end{array}$ & $\begin{array}{l}0.31 \\
0.04 \\
\end{array}$ & $\begin{array}{l}2.64 \\
0.09 \\
\end{array}$ & $\begin{array}{l}87.36 \\
0.22 \\
\end{array}$ & $\begin{array}{l}0.29 \\
0.01 \\
\end{array}$ & $\begin{array}{l}0.55 \\
0.02 \\
\end{array}$ & $\begin{array}{l}7.03 \\
0.06 \\
\end{array}$ & $\begin{array}{l}0.08 \\
0.00 \\
\end{array}$ & $\begin{array}{l}0.30 \\
0.00 \\
\end{array}$ & $\begin{array}{l}0.52 \\
0.01 \\
\end{array}$ & $\begin{array}{l}\mathrm{bd} \\
\mathrm{bd}\end{array}$ & $\begin{array}{l}0.01 \\
0.00 \\
\end{array}$ & $\begin{array}{l}0.05 \\
0.00 \\
\end{array}$ & $\begin{array}{l}0.42 \\
0.02 \\
\end{array}$ & $\begin{array}{l}0.03 \\
0.00 \\
\end{array}$ & $\begin{array}{l}\mathrm{bd} \\
\mathrm{bd}\end{array}$ & $\begin{array}{l}\mathrm{bd} \\
\mathrm{bd}\end{array}$ & $\begin{array}{l}\mathrm{bd} \\
\mathrm{bd}\end{array}$ \\
\hline MG10 & $\begin{array}{l}\text { Avg } \\
\text { std }\end{array}$ & $\begin{array}{l}0.19 \\
0.06\end{array}$ & $\begin{array}{l}0.41 \\
0.08 \\
\end{array}$ & $\begin{array}{l}3.12 \\
0.48 \\
\end{array}$ & $\begin{array}{l}86.54 \\
0.97\end{array}$ & $\begin{array}{l}0.23 \\
0.03 \\
\end{array}$ & $\begin{array}{l}1.19 \\
0.18 \\
\end{array}$ & $\begin{array}{l}6.85 \\
0.16\end{array}$ & $\begin{array}{l}0.09 \\
0.00\end{array}$ & $\begin{array}{l}0.30 \\
0.01 \\
\end{array}$ & $\begin{array}{l}0.56 \\
0.06 \\
\end{array}$ & $\begin{array}{l}\mathrm{bd} \\
\mathrm{bd}\end{array}$ & $\begin{array}{l}0.03 \\
0.01 \\
\end{array}$ & $\begin{array}{l}0.05 \\
0.00 \\
\end{array}$ & $\begin{array}{l}0.39 \\
0.03 \\
\end{array}$ & $\begin{array}{l}0.03 \\
0.00 \\
\end{array}$ & $\begin{array}{l}\mathrm{bd} \\
\mathrm{bd}\end{array}$ & $\begin{array}{l}0.01 \\
0.00\end{array}$ & $\begin{array}{l}\mathrm{bd} \\
\mathrm{bd}\end{array}$ \\
\hline MG11 & $\begin{array}{l}\text { Avg } \\
\text { std }\end{array}$ & $\begin{array}{l}0.92 \\
0.37 \\
\end{array}$ & $\begin{array}{l}0.34 \\
0.05 \\
\end{array}$ & $\begin{array}{l}2.91 \\
0.10 \\
\end{array}$ & $\begin{array}{c}85.70 \\
0.84 \\
\end{array}$ & $\begin{array}{l}0.17 \\
0.03 \\
\end{array}$ & $\begin{array}{l}1.31 \\
0.07 \\
\end{array}$ & $\begin{array}{l}7.29 \\
0.41 \\
\end{array}$ & $\begin{array}{l}0.08 \\
0.01 \\
\end{array}$ & $\begin{array}{l}0.30 \\
0.01 \\
\end{array}$ & $\begin{array}{l}0.51 \\
0.03 \\
\end{array}$ & $\begin{array}{l}\mathrm{bd} \\
\mathrm{bd}\end{array}$ & $\begin{array}{l}0.02 \\
0.00\end{array}$ & $\begin{array}{l}0.05 \\
0.00 \\
\end{array}$ & $\begin{array}{l}0.34 \\
0.02 \\
\end{array}$ & $\begin{array}{l}0.03 \\
0.00 \\
\end{array}$ & $\begin{array}{l}\mathrm{bd} \\
\mathrm{bd} \\
\mathrm{bd}\end{array}$ & $\begin{array}{l}0.01 \\
0.00 \\
\end{array}$ & $\begin{array}{l}\mathrm{bd} \\
\mathrm{bd}\end{array}$ \\
\hline MG12 & $\begin{array}{l}\text { Avg } \\
\text { stdd }\end{array}$ & $\begin{array}{l}0.63 \\
0.06 \\
\end{array}$ & $\begin{array}{l}0.45 \\
0.04 \\
\end{array}$ & $\begin{array}{l}3.48 \\
0.13 \\
\end{array}$ & $\begin{array}{c}85.51 \\
0.76 \\
\end{array}$ & $\begin{array}{l}0.40 \\
0.06 \\
\end{array}$ & $\begin{array}{l}0.88 \\
0.20 \\
\end{array}$ & $\begin{array}{l}7.21 \\
0.23 \\
\end{array}$ & $\begin{array}{l}0.11 \\
0.01 \\
\end{array}$ & $\begin{array}{l}0.34 \\
0.00 \\
\end{array}$ & $\begin{array}{l}0.58 \\
0.02 \\
\end{array}$ & $\begin{array}{l}\mathrm{bd} \\
\mathrm{bd}\end{array}$ & $\begin{array}{l}0.01 \\
0.00 \\
\end{array}$ & $\begin{array}{l}0.05 \\
0.00 \\
\end{array}$ & $\begin{array}{l}0.29 \\
0.01 \\
\end{array}$ & $\begin{array}{l}0.04 \\
0.00 \\
\end{array}$ & $\begin{array}{l}\mathrm{bd} \\
\mathrm{bd}\end{array}$ & $\begin{array}{l}\mathrm{bd} \\
\mathrm{bd}\end{array}$ & $\begin{array}{l}\mathrm{bd} \\
\mathrm{bd}\end{array}$ \\
\hline MG14 & $\begin{array}{l}\text { Avg } \\
\text { std }\end{array}$ & $\begin{array}{l}0.97 \\
0.14\end{array}$ & $\begin{array}{l}0.94 \\
0.12\end{array}$ & $\begin{array}{l}2.98 \\
0.20\end{array}$ & $\begin{array}{c}83.44 \\
0.12\end{array}$ & $\begin{array}{l}0.23 \\
0.02\end{array}$ & $\begin{array}{l}0.96 \\
0.02\end{array}$ & $\begin{array}{l}8.05 \\
0.12\end{array}$ & $\begin{array}{l}0.19 \\
0.02\end{array}$ & $\begin{array}{l}0.80 \\
0.01\end{array}$ & $\begin{array}{l}0.85 \\
0.00\end{array}$ & $\begin{array}{l}0.01 \\
0.00\end{array}$ & $\begin{array}{l}0.01 \\
0.00\end{array}$ & $\begin{array}{l}0.07 \\
0.01\end{array}$ & $\begin{array}{l}0.38 \\
0.04\end{array}$ & $\begin{array}{l}0.08 \\
0.00\end{array}$ & $\begin{array}{l}0.01 \\
0.01\end{array}$ & $\begin{array}{l}\mathrm{bd} \\
\mathrm{bd}\end{array}$ & $\begin{array}{l}\mathrm{bd} \\
\mathrm{bd}\end{array}$ \\
\hline MG15 & $\begin{array}{l}\text { Avg } \\
\text { std }\end{array}$ & $\begin{array}{l}0.44 \\
0.21 \\
\end{array}$ & $\begin{array}{l}0.32 \\
0.02 \\
\end{array}$ & $\begin{array}{l}2.99 \\
0.10 \\
\end{array}$ & $\begin{array}{c}86.93 \\
0.50 \\
\end{array}$ & $\begin{array}{l}0.26 \\
0.06 \\
\end{array}$ & $\begin{array}{l}1.02 \\
0.16 \\
\end{array}$ & $\begin{array}{l}6.64 \\
0.12 \\
\end{array}$ & $\begin{array}{l}0.09 \\
0.02 \\
\end{array}$ & $\begin{array}{l}0.28 \\
0.02\end{array}$ & $\begin{array}{l}0.61 \\
0.14\end{array}$ & $\begin{array}{l}\mathrm{bd} \\
\mathrm{bd}\end{array}$ & $\begin{array}{l}0.01 \\
0.00\end{array}$ & $\begin{array}{l}0.05 \\
0.00\end{array}$ & $\begin{array}{l}0.28 \\
0.05\end{array}$ & $\begin{array}{l}0.03 \\
0.00\end{array}$ & $\begin{array}{l}\mathrm{bd} \\
\mathrm{bd}\end{array}$ & $\begin{array}{l}0.01 \\
0.00\end{array}$ & $\begin{array}{l}\mathrm{bd} \\
\mathrm{bd}\end{array}$ \\
\hline MG16 & $\begin{array}{l}\text { Avg } \\
\text { std }\end{array}$ & $\begin{array}{l}0.70 \\
0.13 \\
\end{array}$ & $\begin{array}{l}0.36 \\
0.12 \\
\end{array}$ & $\begin{array}{l}2.88 \\
0.07 \\
\end{array}$ & $\begin{array}{c}87.50 \\
0.38 \\
\end{array}$ & $\begin{array}{l}0.32 \\
0.02 \\
\end{array}$ & $\begin{array}{l}0.58 \\
0.10 \\
\end{array}$ & $\begin{array}{l}6.35 \\
0.15 \\
\end{array}$ & $\begin{array}{l}0.08 \\
0.00 \\
\end{array}$ & $\begin{array}{l}0.29 \\
0.00 \\
\end{array}$ & $\begin{array}{l}0.52 \\
0.01 \\
\end{array}$ & $\begin{array}{l}\mathrm{bd} \\
\mathrm{bd}\end{array}$ & $\begin{array}{l}0.01 \\
0.00 \\
\end{array}$ & $\begin{array}{l}0.05 \\
0.00 \\
\end{array}$ & $\begin{array}{l}0.31 \\
0.01 \\
\end{array}$ & $\begin{array}{l}0.03 \\
0.00 \\
\end{array}$ & $\begin{array}{l}\mathrm{bd} \\
\mathrm{bd}\end{array}$ & $\begin{array}{l}\mathrm{bd} \\
\mathrm{bd}\end{array}$ & $\begin{array}{l}\mathrm{bd} \\
\mathrm{bd}\end{array}$ \\
\hline MG17 & $\begin{array}{l}\text { Avg } \\
\text { std }\end{array}$ & $\begin{array}{l}1.38 \\
0.17\end{array}$ & $\begin{array}{l}0.42 \\
0.04\end{array}$ & $\begin{array}{l}3.61 \\
0.36 \\
\end{array}$ & $\begin{array}{l}83.59 \\
0.24\end{array}$ & $\begin{array}{l}0.22 \\
0.02\end{array}$ & $\begin{array}{l}0.91 \\
0.02 \\
\end{array}$ & $\begin{array}{l}8.01 \\
0.15\end{array}$ & $\begin{array}{l}0.10 \\
0.01\end{array}$ & $\begin{array}{l}0.51 \\
0.04 \\
\end{array}$ & $\begin{array}{l}0.86 \\
0.06\end{array}$ & $\begin{array}{l}\text { bd } \\
\text { bd }\end{array}$ & $\begin{array}{l}0.01 \\
0.00 \\
\end{array}$ & $\begin{array}{l}0.06 \\
0.00 \\
\end{array}$ & $\begin{array}{l}0.21 \\
0.03 \\
\end{array}$ & $\begin{array}{l}0.08 \\
0.00 \\
\end{array}$ & $\begin{array}{l}\text { bd } \\
\text { bd }\end{array}$ & $\begin{array}{l}\text { bd } \\
\text { bd }\end{array}$ & $\begin{array}{l}\mathrm{bd} \\
\mathrm{bd}\end{array}$ \\
\hline MG18 & $\begin{array}{l}\text { Avg } \\
\text { stdd }\end{array}$ & $\begin{array}{l}0.58 \\
0.26 \\
\end{array}$ & $\begin{array}{l}0.38 \\
0.06 \\
\end{array}$ & $\begin{array}{l}2.93 \\
0.05 \\
\end{array}$ & $\begin{array}{c}86.16 \\
0.58 \\
\end{array}$ & $\begin{array}{l}0.32 \\
0.02 \\
\end{array}$ & $\begin{array}{l}0.71 \\
0.04 \\
\end{array}$ & $\begin{array}{l}7.55 \\
0.16 \\
\end{array}$ & $\begin{array}{l}0.09 \\
0.00\end{array}$ & $\begin{array}{l}0.32 \\
0.01 \\
\end{array}$ & $\begin{array}{l}0.61 \\
0.02 \\
\end{array}$ & $\begin{array}{l}\mathrm{bd} \\
\mathrm{bd}\end{array}$ & $\begin{array}{l}0.01 \\
0.00 \\
\end{array}$ & $\begin{array}{l}0.05 \\
0.00 \\
\end{array}$ & $\begin{array}{l}0.24 \\
0.04 \\
\end{array}$ & $\begin{array}{l}0.04 \\
0.00 \\
\end{array}$ & $\begin{array}{l}\mathrm{bd} \\
\mathrm{bd}\end{array}$ & $\begin{array}{l}\mathrm{bd} \\
\mathrm{bd}\end{array}$ & $\begin{array}{l}\mathrm{bd} \\
\mathrm{bd}\end{array}$ \\
\hline MG19 & $\begin{array}{l}\text { Avg } \\
\text { stdd }\end{array}$ & $\begin{array}{l}0.41 \\
0.13 \\
\end{array}$ & $\begin{array}{l}0.23 \\
0.09 \\
\end{array}$ & $\begin{array}{l}2.99 \\
0.19 \\
\end{array}$ & $\begin{array}{c}88.68 \\
0.72 \\
\end{array}$ & $\begin{array}{l}0.15 \\
0.03 \\
\end{array}$ & $\begin{array}{l}1.02 \\
0.08 \\
\end{array}$ & $\begin{array}{l}5.23 \\
0.17 \\
\end{array}$ & $\begin{array}{l}0.07 \\
0.00 \\
\end{array}$ & $\begin{array}{l}0.26 \\
0.00 \\
\end{array}$ & $\begin{array}{l}0.45 \\
0.02 \\
\end{array}$ & $\begin{array}{l}\mathrm{bd} \\
\mathrm{bd}\end{array}$ & $\begin{array}{l}0.02 \\
0.00 \\
\end{array}$ & $\begin{array}{l}0.05 \\
0.00 \\
\end{array}$ & $\begin{array}{l}0.40 \\
0.02 \\
\end{array}$ & $\begin{array}{l}0.02 \\
0.00 \\
\end{array}$ & $\begin{array}{l}\text { bd } \\
\text { bd }\end{array}$ & $\begin{array}{l}0.01 \\
0.00 \\
\end{array}$ & $\begin{array}{l}\mathrm{bd} \\
\mathrm{bd}\end{array}$ \\
\hline MG20 & $\begin{array}{l}\text { Avg } \\
\text { std }\end{array}$ & $\begin{array}{l}17.46 \\
0.11\end{array}$ & $\begin{array}{l}0.16 \\
0.05\end{array}$ & $\begin{array}{l}2.24 \\
0.03\end{array}$ & $\begin{array}{c}71.04 \\
0.20\end{array}$ & $\begin{array}{l}0.17 \\
0.03\end{array}$ & $\begin{array}{l}0.55 \\
0.01\end{array}$ & $\begin{array}{l}6.71 \\
0.13\end{array}$ & $\begin{array}{l}0.07 \\
0.00\end{array}$ & $\begin{array}{l}0.35 \\
0.01\end{array}$ & $\begin{array}{l}0.74 \\
0.02\end{array}$ & $\begin{array}{l}\mathrm{bd} \\
\mathrm{bd}\end{array}$ & $\begin{array}{l}0.01 \\
0.00\end{array}$ & $\begin{array}{l}0.04 \\
0.00\end{array}$ & $\begin{array}{l}0.39 \\
0.02\end{array}$ & $\begin{array}{l}0.03 \\
0.00\end{array}$ & $\begin{array}{l}\mathrm{bd} \\
\mathrm{bd}\end{array}$ & $\begin{array}{l}\mathrm{bd} \\
\mathrm{bd}\end{array}$ & $\begin{array}{l}\mathrm{bd} \\
\mathrm{bd}\end{array}$ \\
\hline
\end{tabular}


Table 6. Chemical composition (SEM-EDS, wt\% oxides, normalised) of the cross-sections of unmelted (UG2, UG3, and UG4) and melted (MG7, MG15, and MG19) fragments ( $b d=$ below detection).

\begin{tabular}{|c|c|c|c|c|c|c|c|c|c|c|c|c|c|c|c|c|c|c|}
\hline \multicolumn{2}{|c|}{ Sample } & \multirow{3}{*}{$\begin{array}{c}\mathbf{N a}_{\mathbf{2}} \mathbf{O} \\
17.39 \\
1.39\end{array}$} & \multirow{3}{*}{$\begin{array}{c}\text { MgO } \\
0.55 \\
0.15\end{array}$} & \multirow{3}{*}{$\begin{array}{c}\mathbf{A l}_{\mathbf{2}} \mathbf{O}_{\mathbf{3}} \\
2.25 \\
0.07\end{array}$} & \multirow{3}{*}{$\begin{array}{c}\mathrm{SiO}_{2} \\
69.53 \\
1.00\end{array}$} & \multirow{3}{*}{$\begin{array}{l}\mathbf{S O}_{3} \\
0.10 \\
0.15\end{array}$} & \multirow{3}{*}{$\begin{array}{c}\mathbf{K}_{\mathbf{2}} \mathbf{O} \\
0.61 \\
0.04\end{array}$} & \multirow{3}{*}{$\begin{array}{l}\mathrm{CaO} \\
7.27 \\
0.50\end{array}$} & \multirow{3}{*}{$\begin{array}{c}\mathrm{TiO}_{2} \\
0.13 \\
0.04\end{array}$} & \multirow{3}{*}{$\begin{array}{c}\mathbf{M n O}_{\mathbf{2}} \\
0.31 \\
0.06\end{array}$} & \multirow{3}{*}{$\begin{array}{c}\mathbf{F e}_{2} \mathbf{O}_{3} \\
0.87 \\
0.11\end{array}$} & \multirow{3}{*}{$\begin{array}{c}\mathrm{CoO} \\
0.08 \\
0.03\end{array}$} & \multirow{3}{*}{$\begin{array}{c}\mathrm{CuO} \\
0.02 \\
0.03\end{array}$} & \multirow{3}{*}{$\begin{array}{l}\text { SrO } \\
0.24 \\
0.13\end{array}$} & \multirow{3}{*}{$\begin{array}{c}\mathbf{S b}_{\mathbf{2}} \mathbf{O}_{\mathbf{5}} \\
0.30 \\
0.35\end{array}$} & \multirow{3}{*}{$\begin{array}{c}\mathbf{P b O} \\
0.07 \\
0.03\end{array}$} & \multirow{3}{*}{$\begin{array}{c}\mathbf{P}_{\mathbf{2}} \mathbf{O}_{5} \\
0.04 \\
0.06\end{array}$} & \multirow{3}{*}{$\begin{array}{c}\mathrm{SnO}_{2} \\
0.22 \\
0.03\end{array}$} \\
\hline & Avg & & & & & & & & & & & & & & & & & \\
\hline UG2 bulk & std & & & & & & & & & & & & & & & & & \\
\hline \multirow{2}{*}{ UG2 surface } & Avg & 18.14 & 0.63 & 2.36 & 69.20 & 0.15 & 0.60 & 7.04 & 0.08 & 0.28 & 0.88 & $\mathrm{bd}$ & 0.18 & 0.15 & $\mathrm{bd}$ & 0.23 & 0.07 & $\mathrm{bd}$ \\
\hline & std & 1.10 & 0.13 & 0.08 & 0.83 & 0.06 & 0.04 & 0.96 & 0.04 & 0.04 & 0.21 & bd & 0.10 & 0.22 & bd & 0.25 & 0.10 & bd \\
\hline \multirow[b]{2}{*}{ UG3 bulk } & Avg & 17.38 & 0.43 & 2.32 & 69.54 & 0.31 & 0.63 & 6.85 & 0.02 & 0.62 & 0.53 & 0.01 & 0.07 & 0.22 & 0.55 & 0.15 & 0.13 & 0.22 \\
\hline & std & 2.16 & 0.18 & 0.13 & 2.22 & 0.08 & 0.00 & 0.31 & 0.03 & 0.08 & 0.18 & 0.02 & 0.05 & 0.08 & 0.44 & 0.21 & 0.16 & 0.32 \\
\hline \multirow{2}{*}{ UG3 surface } & Avg & 16.19 & 0.49 & 2.33 & 70.77 & 0.36 & 0.67 & 7.14 & 0.10 & 0.63 & 0.59 & 0.05 & 0.04 & 0.10 & 0.16 & 0.18 & 0.09 & 0.14 \\
\hline & std & 1.29 & 0.05 & 0.08 & 0.32 & 0.00 & 0.04 & 0.66 & 0.01 & 0.21 & 0.12 & 0.06 & 0.05 & 0.13 & 0.04 & 0.07 & 0.04 & 0.20 \\
\hline UG4 bulk & std & 2.51 & 0.10 & 0.06 & 1.61 & 0.11 & 0.06 & 0.21 & 0.10 & 0.17 & 0.06 & 0.03 & 0.07 & 0.13 & 0.29 & 0.16 & 0.14 & 0.03 \\
\hline \multirow{2}{*}{ UG4 surface } & Avg & 13.51 & 0.52 & 2.51 & 71.73 & 0.21 & 1.00 & 7.63 & 0.20 & 0.57 & 0.70 & 0.07 & 0.14 & 0.16 & 0.18 & 0.32 & 0.19 & 0.40 \\
\hline & std & 0.28 & 0.00 & 0.29 & 0.52 & 0.05 & 0.21 & 0.12 & 0.03 & 0.00 & 0.09 & 0.06 & 0.12 & 0.22 & 0.21 & 0.45 & 0.02 & 0.06 \\
\hline \multirow{2}{*}{ MG7 bulk } & Avg & 13.76 & 0.64 & 2.04 & 72.06 & 0.36 & 0.80 & 7.67 & 0.26 & 0.31 & 0.59 & $\mathrm{bd}$ & 0.10 & 0.08 & 0.66 & 0.40 & $\mathrm{bd}$ & 0.27 \\
\hline & std & 0.23 & 0.11 & 0.22 & 0.37 & 0.11 & 0.03 & 0.42 & 0.23 & 0.12 & 0.12 & $\mathrm{bd}$ & 0.09 & 0.37 & 0.25 & 0.35 & $\mathrm{bd}$ & 0.32 \\
\hline \multirow[b]{2}{*}{ MG7 surface } & Avg & 2.57 & 1.90 & 14.64 & 58.01 & $\mathrm{bd}$ & 1.42 & 7.43 & $\mathrm{bd}$ & 1.28 & 8.63 & $\mathrm{bd}$ & $\mathrm{bd}$ & 1.56 & $\mathrm{bd}$ & 1.55 & 1.00 & $\mathrm{bd}$ \\
\hline & std & 2.13 & 0.64 & 5.02 & 5.20 & bd & 0.04 & 1.20 & bd & 0.18 & 0.76 & bd & bd & 1.50 & bd & 0.06 & 0.13 & bd \\
\hline MG15 bulk & Avg & 16.83 & 0.54 & 2.31 & 68.94 & 0.26 & 1.53 & 6.52 & 0.19 & 0.32 & 0.62 & 0.07 & $\mathrm{bd}$ & 0.56 & 0.46 & 0.21 & 0.29 & 0.37 \\
\hline \multirow[b]{2}{*}{ MG15 surface } & Avg & 0.41 & 0.35 & 2.43 & 81.41 & 0.17 & 1.32 & 9.87 & 0.32 & 0.82 & 1.01 & $\mathrm{bd}$ & $\mathrm{bd}$ & 0.83 & 0.66 & 0.30 & 0.10 & $\mathrm{bd}$ \\
\hline & std & 0.13 & 0.00 & 0.13 & 0.28 & 0.03 & 0.06 & 2.38 & 0.16 & 0.49 & 0.43 & bd & bd & 0.32 & 0.01 & 0.23 & 0.00 & bd \\
\hline & Avg & 17.94 & 0.61 & 2.26 & 69.69 & 0.27 & 0.70 & 6.72 & 0.07 & 0.23 & 0.43 & 0.03 & 0.08 & 0.26 & 0.25 & 0.24 & 0.11 & 0.09 \\
\hline MG19 bulk & std & 2.39 & 0.12 & 0.08 & 2.14 & 0.08 & 0.14 & 0.35 & 0.01 & 0.02 & 0.10 & 0.04 & 0.06 & 0.37 & 0.19 & 0.34 & 0.16 & 0.13 \\
\hline & Avg & 0.31 & 0.80 & 2.96 & 85.36 & 0.37 & 0.61 & 7.12 & 0.03 & 0.31 & 0.81 & 0.14 & 0.04 & 0.07 & 0.61 & 0.08 & 0.17 & 0.22 \\
\hline MG19 surface & std & 0.27 & 0.22 & 0.12 & 1.34 & 0.01 & 0.76 & 0.62 & 0.02 & 0.01 & 0.24 & 0.01 & 0.06 & 0.10 & 0.13 & 0.11 & 0.17 & 0.30 \\
\hline
\end{tabular}


In Roman glass both the aluminium and calcium are introduced mainly with the silica source and this can be investigated by plotting the $\mathrm{CaO}$ and $\mathrm{Al}_{2} \mathrm{O}_{3}$ content (Figure $2 \mathrm{~b}$ ), determined for the surface using XRF. Except for the light green bead (B5, light green triangle), the concentration of $\mathrm{Al}_{2} \mathrm{O}_{3}$ in the assemblage is comparable. Much Roman glass was made using white beach sand, which contained calcium-rich shells but was otherwise quite a pure source of silica [23,24].

\subsection{The Colour of the Glass}

The Roman glass samples show a wide range of blue-green hues. The transparent pale blue or green colour of most of the vessel glass is due to small quantities of iron impurities introduced to the glass with the raw materials. This colour was often adjusted by adding manganese or antimony compounds, which could modify or completely neutralise the colour. Figure $3 \mathrm{a}$ shows the $\mathrm{Fe}_{2} \mathrm{O}_{3}$ and $\mathrm{MnO}$ content of the glass samples, measured at the surface by XRF. Based on other published glass assemblages, the dotted line divides colourless (on the left) and coloured (on the right) glass [16]: the colourless glass tends to contain a high ratio of manganese to iron whereas the strongly blue and green glass and beads have a high concentration of $\mathrm{Fe}_{2} \mathrm{O}_{3}(>1 \mathrm{wt} \%)$. All of the Birdoswald glass falls into the blue-green composition field.
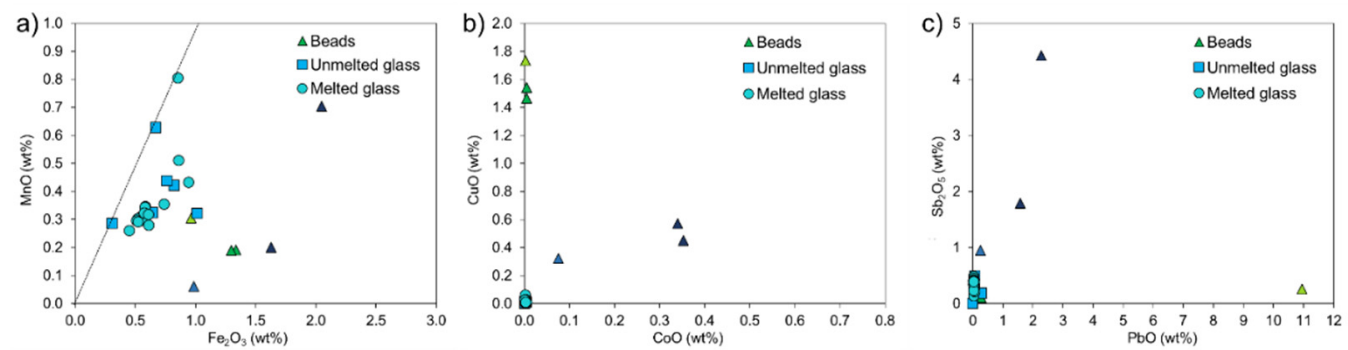

Figure 3. XRF data for the glass surface, showing $\mathrm{Fe}_{2} \mathrm{O}_{3}$ and $\mathrm{MnO}(\mathbf{a}), \mathrm{CoO}$ and $\mathrm{CuO}(\mathbf{b})$, and $\mathrm{PbO}$ and $\mathrm{Sb}_{2} \mathrm{O}_{5}(\mathbf{c})$ in the glass fragments.

Dark blue and green glass beads are also present in the assemblage, and they were formed by intentionally adding colourants and opacifiers. Cobalt oxide is considered as the chromophore responsible for the blue colour of the glass beads (Figure 3b). The nature of the cobalt colorant used in glass is not fully understood. Different researchers proved consistent positive correlation between iron and cobalt oxides in blue glass, and this is confirmed as well in this study, suggesting the use of a mineral cobalt source with a similar composition to those reported in the literature [22,25]. Our results also indicate a correlation between cobalt, manganese, copper, and lead oxide, which are probably trace elements associated with cobalt minerals (Table 3) [22,26].

The green glass beads contain a high amount of iron and copper oxides, which were used to impart the green colour (Figure 3). The presence of copper, lead and tin in the glass samples indicates that oxidised copper alloys (bronze) were added to the melted glass and employed as chromophores [22,27-30].

The antimony oxide in the transparent blue bead (B1, light blue triangle) is in solution, and has the role of decolourising agent in the colourless glass base; however, some of the beads contain antimony-rich opacifiers: tiny crystals that scatter light and make the glass opaque. In order to evaluate the type of opacifier introduced in the formulation, the $\mathrm{PbO}$ and $\mathrm{Sb}_{2} \mathrm{O}_{5}$ content was plotted in Figure $3 \mathrm{c}$, again based on the XRF surface analyses. Except for the blue bead and fragments, most of the samples have a low concentration of $\mathrm{Sb}_{2} \mathrm{O}_{3}(<0.5 \mathrm{wt} \%)$. In the opaque dark blue glass fragments (B2 and B3, dark blue triangles), the higher concentration of $\mathrm{Sb}_{2} \mathrm{O}_{3}$ can be explained by the use of calcium antimonate compounds $\left(\mathrm{Ca}_{2} \mathrm{Sb}_{2} \mathrm{O}_{7}\right.$ or $\left.\mathrm{CaSb}_{2} \mathrm{O}_{6}\right)$ as opacifier $[28,29]$. The higher concentration of $\mathrm{PbO}$ in the opaque dark blue fragments compared to the other glass can be related to the cobaltbased colourant used to create the blue colour (Figure 3). The opaque light green bead (B5, 
light green triangle) exhibits a very high concentration of $\mathrm{PbO}$ (Figure 3c) as a result of the use of lead antimonate $\left(\mathrm{Pb}_{2} \mathrm{Sb}_{2} \mathrm{O}_{7}\right)$ as a yellow opacifier. Green glass was manufactured by using yellow lead antimonate crystals and copper oxide dissolved in the glass [28,29]. It was previously reported that tin oxide is sometimes present in aggregates together with lead antimonate crystals in Roman glasses [28]. This would explain the higher concentration of tin oxide in the opaque light green beads compared to the transparent green beads (Table 3).

\section{Discussion}

The main group of Roman glass fragments consists of transparent natron glass with a range of blue-green hues. Blue-green is the most common colour of vessels in the 1 st-3rd centuries, it was quite common also until the 4th century, and it was used for bottles, household containers, and higher quality tablewares [15]. In the 1st and 2nd centuries, large glass vessels and jars were reused in burials to contain ashes. Small unguent bottles containing scented oils, pastes and powders were frequent in both cremation and inhumation burials in the 1st and 3rd centuries [15].

\subsection{Recycled Glass}

The Roman glass fragments and beads are representative of three compositional groups [16]: antimony colourless (Sb), manganese (Mn), and mixed antimony and manganese (Sb-Mn) (Table 2). Antimony glass is thought to derive from Egypt and manganese glass probably was made in the Levant, so fresh glass should contain either $\mathrm{Sb}$ or Mn but not both, whereas glass containing both ( $\mathrm{Sb}-\mathrm{Mn})$ is recycled [31].

The base of the transparent blue bead (B1, light blue triangle) is an antimony glass, which was common in assemblages from the 1st to 3rd centuries, and it was produced using sands without impurities, adding antimony as a decolouriser [16] (Figure 4 and Table 3).
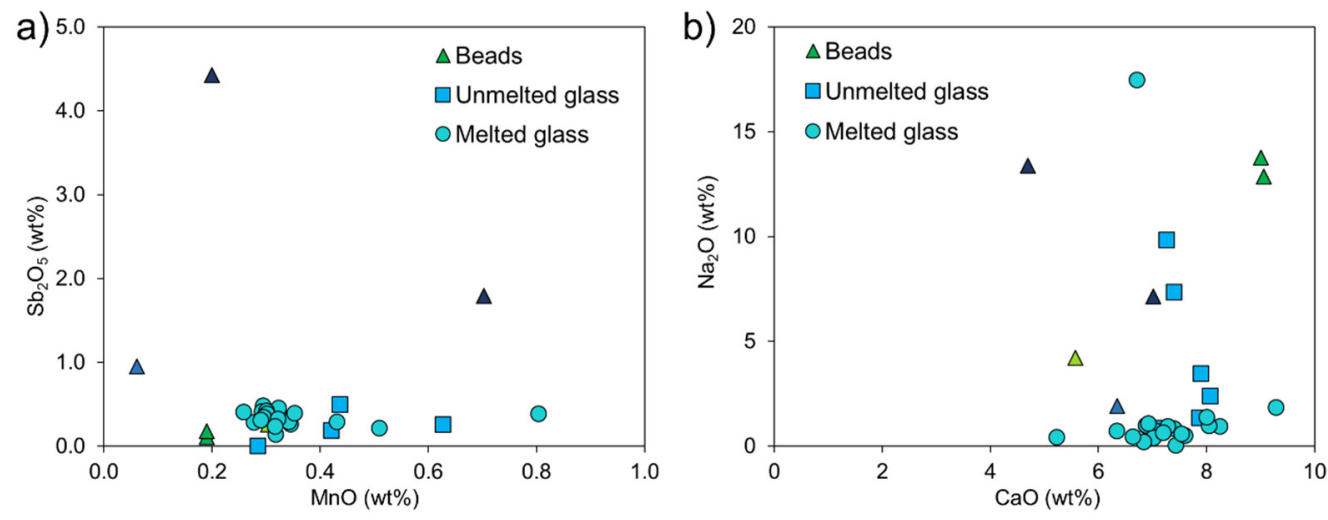

Figure 4. XRF data for the glass surface. Correlation of $\mathrm{MnO}$ and $\mathrm{Sb}_{2} \mathrm{O}_{5}(\mathbf{a})$, and $\mathrm{CaO}$ and $\mathrm{Na}_{2} \mathrm{O}$ (b) in the glass fragments.

The only representative of the low-manganese group is a blue-green fragment (UG1), as it contains low $\mathrm{MnO}(\mathrm{wt} \%)$ and no antimony (Figure 4 and Table 4$)$. This group of glass originates from the Syria-Palestine region, and it was the main type of fresh blue-green glass by the 1st century [16].

The largest group represented in the Birdoswald assemblage is Sb-Mn glass. The glass contains manganese and antimony, it is the product of the recycling of manganese and antimony glass, and it became the main group of blue-green glass in Britain in the 2nd and 3rd centuries (Figure 4 and Tables 3-5). The difference in the blue-green tints is largely due to the concentrations of antimony, manganese, and iron oxide. Colourless glass was used for higher quality objects and was commonly recycled. However, after repeated re-melting, the colourless glass tends to absorb different contaminants, resulting in a blue-green hue [16]. 


\subsection{Sodium Depletion}

In Figure 4, the concentrations of $\mathrm{CaO}$ and $\mathrm{Na}_{2} \mathrm{O}$ in the glass samples determined by $\mathrm{XRF}$ of the surface are plotted, showing that the glass assemblage exhibits a wide range of $\mathrm{Na}_{2} \mathrm{O}$ concentration at the surface of the fragments. The $\mathrm{Na}_{2} \mathrm{O}$ content in some of the glass is far too low for a glass to form at achievable temperatures, and therefore is due to the depletion of sodium oxide from the surface due to surface degradation; this is especially evident for the melted glass samples. Glass is very prone to weathering agents like water in archaeological burial, and some elements, in particular soda, can be leached from the surface, which becomes richer in other elements, like silica [32-34]. The higher depletion of sodium in the melted glass fragments may be due to the evaporation of sodium during the exposure to high temperatures during the cremation ritual $[35,36]$.

To confirm the depletion of soda from the glass surface, SEM observations and elemental analyses were carried out on cross-sections to study the composition of the unaltered glass in the bulk and compare this with the composition at the surface. In the unmelted glass fragments (UG2, UG3, and UG4), the chemical composition of the surface (within 100 $\mu \mathrm{m}$ ) is similar to the bulk, except for a slight decrease in the sodium oxide content (Table 6). The results obtained by XRF on these samples show a lower content in soda compared to SEM-EDS data. This is due to the fact that the signal collected by XRF comes from thin surface layers of weathered areas of the fragments. The melted glass samples (MG7, MG15, and MG19) exhibit significant depletion of soda on the surface (sodium oxide content of about $1 \mathrm{wt} \%$ ), compared to the bulk (sodium oxide content of about $15 \mathrm{wt} \%$ ) (Table 6). From the morphological point of view, the unmelted glass fragments are very homogenous, with smooth surfaces, and no bubbles, phase separation, particles or visible surface weathering layers (Figure 5a). On the contrary, the melted glass samples display round voids and clumps of particles concentrated within $500 \mu \mathrm{m}$ from the surface (Figure $5 \mathrm{~b}-\mathrm{f}$ ). The surface voids are sometimes filled with small particles, probably ashes and soil from the cremation pit (Figure 6). The presence of these round voids and clumps is probably the result of gas release and bubble formation in the glass due to reactions with surrounding ash and debris as the glass was reheated during the cremation process. By reheating the glass, sodium oxide migrates to the surface and it is lost by volatilization. During cooling of the glass, phase separation takes place, and some of the voids can entrap other particles from the soil. This altered surface, with its greater surface area, may then be more susceptible to post-burial weathering, as it can act as hotspots where moisture can concentrate. Depletion of sodium occurs on the surface of the samples and along cracks, where a very thin layer rich in silica but poor in sodium oxide can be observed (Figures $5 f$ and 7 ). These silica-rich areas are formed as a result of the exposure to moisture in the burial environment, which fosters the leaching out of sodium oxide by dissolution [37]. The melted glass fragments have far more severe sodium depletion compared to the unmelted ones, therefore their reheating during the cremation is the main cause of sodium depletion, rather than the burial environment (soil composition, $\mathrm{pH}$ ). Indeed, the exposure to high temperatures increased the rate of the volatilisation reaction, leading to the reduction of the concentration of soda in the surface layer [36].

\subsection{Estimating the Temperature Experienced by the Glass}

A large proportion of the glass has been heated during cremation, enough that it has started to distort, as most was probably positioned closer to the fire. Determining the behaviour of this Roman glass composition at different temperatures can provide information about the temperatures achieved during cremation. To evaluate the melting, stiffening, and gathering temperatures of the glass, the Lakatos model was used [19]. In this model, the viscosity values indicate the temperatures that are necessary to remelt raw glass and then to work the glass. Due to the surface depletion of sodium oxide due to heating followed by weathering, the model used the SEM-EDS data obtained by targeting areas of unaltered glass; for all of these samples the $\mathrm{Na}_{2} \mathrm{O}$ content was consistent with literature data on typical Roman glass (Table 6). 


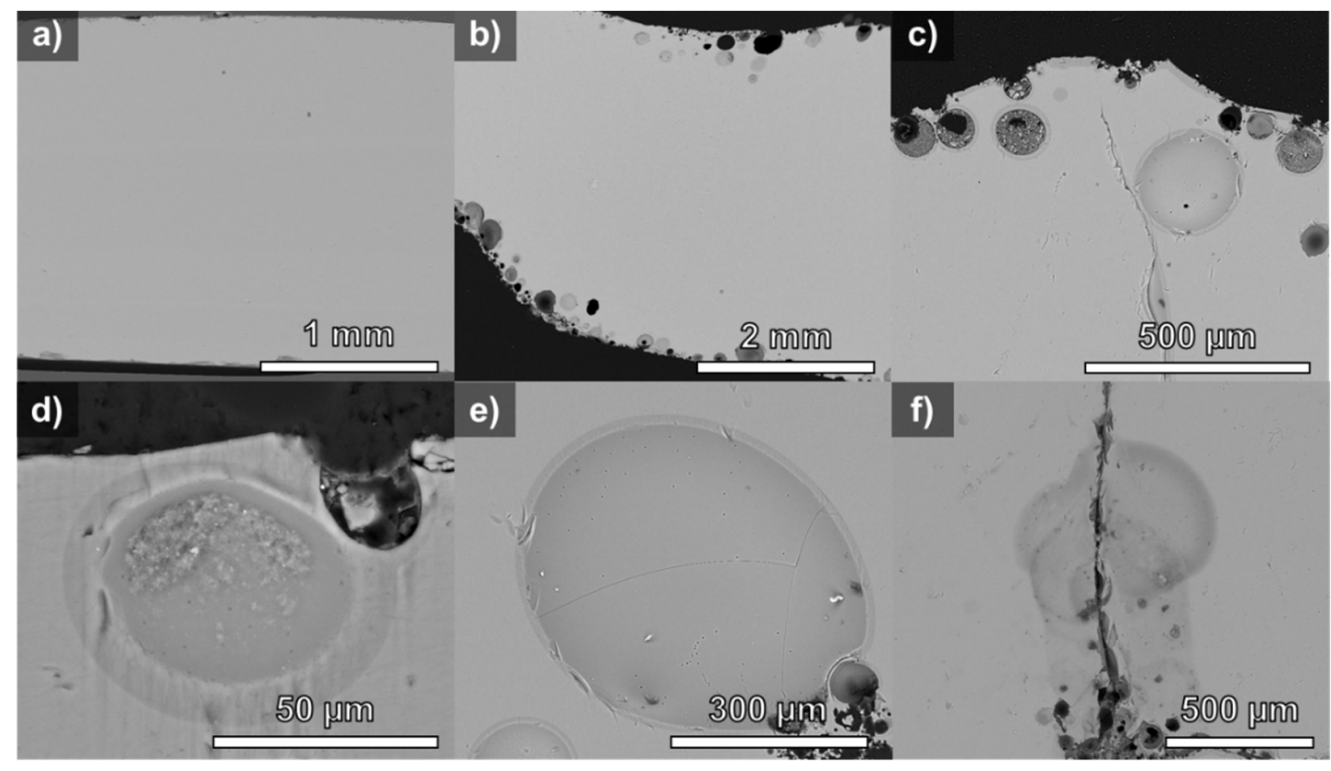

Figure 5. SEM images (BSE detector) of unmelted glass UG4 with a smooth surface (a) and melted glass cross-sections with bubbles, trapped particles and sometimes a visible alteration layer at the surface: MG19 (b,f), MG15 (c,e), and MG7 (d).
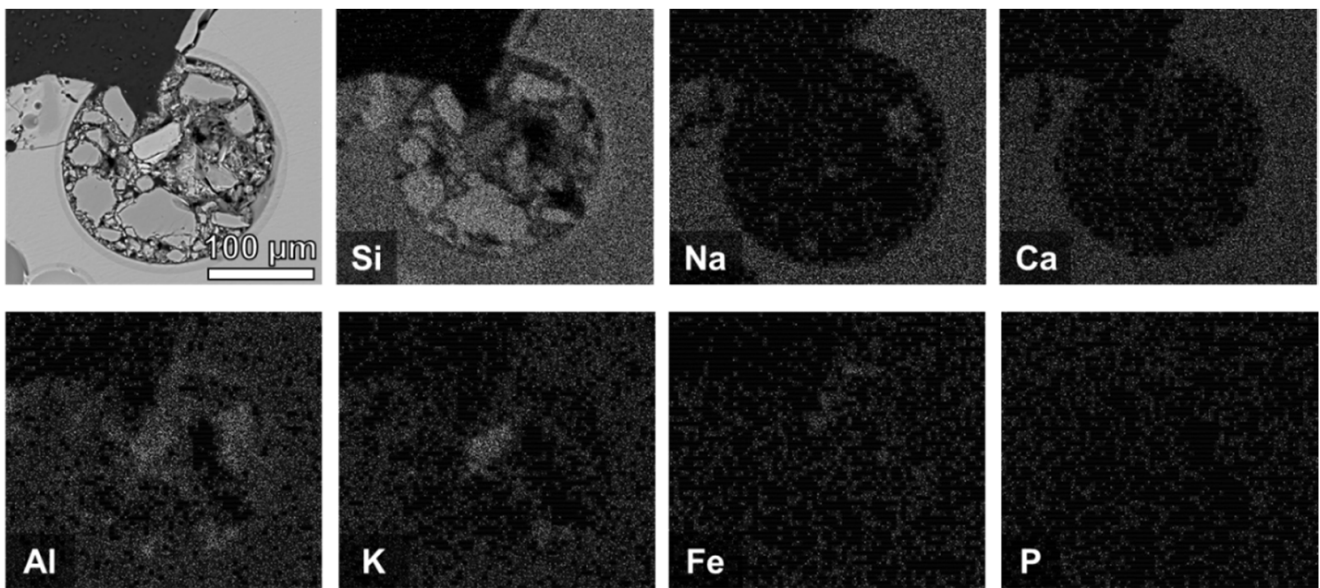

Figure 6. SEM images (BSE detector) and elemental maps of melted glass MG19 cross-section, showing a round area filled with silica and soil particles from the burial pit.
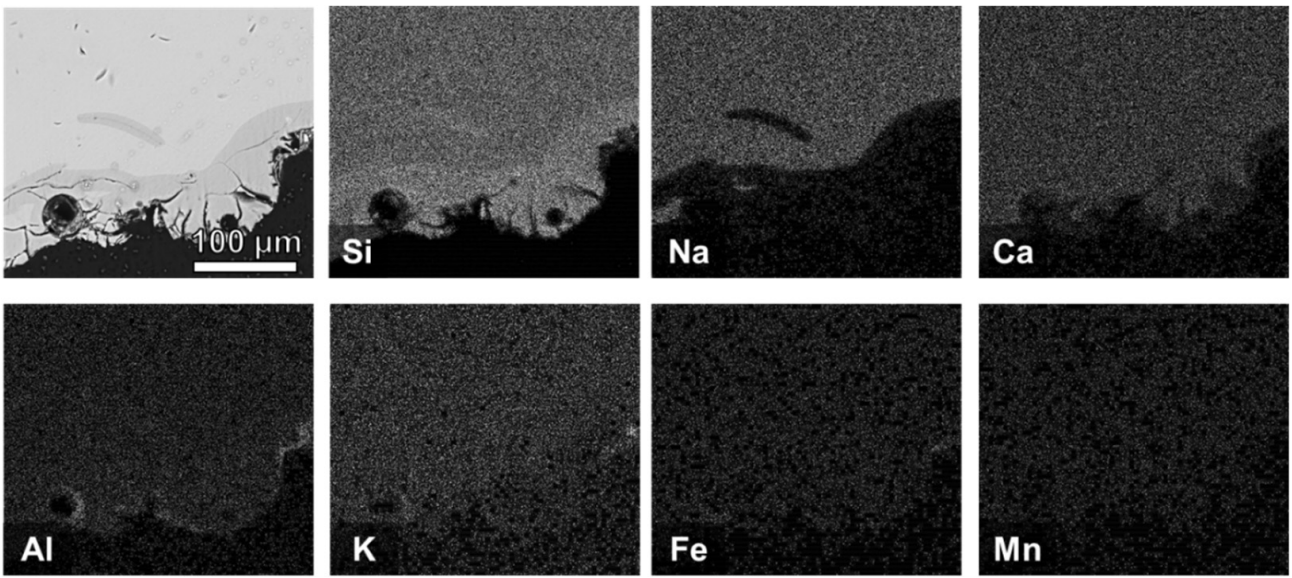

Figure 7. SEM images (BSE detector) and elemental maps of melted glass MG19 cross-section, showing a surface layer rich in silica and depleted of soda. 
According to the model used, the melting temperature range for this glass is about $1200-1230{ }^{\circ} \mathrm{C}$, the gathering temperature reaches about $1120-1150{ }^{\circ} \mathrm{C}$, and the stiffening temperature range is about $685-705{ }^{\circ} \mathrm{C}$ (Table 7). As the Birdoswald glass had softened, but not completely melted, the temperature values intermediate between the stiffening and gathering temperatures (about $695-1135^{\circ} \mathrm{C}$ ) are probably best indicative of the temperatures reached by some of the fragments during the cremation. The results obtained are consistent with data collected by remelting other examples of blue-green Roman glass, which report working temperature ranges of about $660-970{ }^{\circ} \mathrm{C}$ [21]. Some of the glass fragments are badly distorted whereas others are hardly affected; this range is due to the large number of other variables involved in the cremation process, including the position of the objects in the pyre and the length of time that the pyre burned, as well as factors such as the type of vessel (and so the surface area to volume ratio of the glass), and environmental conditions during the ritual.

Table 7. Melting, stiffening, and gathering temperatures of the glass, calculated from the SEM-EDS analyses of the chemical composition using mathematical modelling of the high temperature viscosity of glass.

\begin{tabular}{|c|c|c|c|}
\hline Sample & $\begin{array}{c}\text { Melting } \\
\text { Temperature }{ }^{\circ} \mathrm{C}\end{array}$ & $\begin{array}{c}\text { Gathering } \\
\text { Temperature }{ }^{\circ} \mathrm{C}\end{array}$ & $\begin{array}{c}\text { Stiffening } \\
\text { Temperature }{ }^{\circ} \mathrm{C}\end{array}$ \\
\hline MG7 & 1230 & 1148 & 706 \\
\hline MG15 & 1211 & 1128 & 688 \\
\hline MG19 & 1201 & 1120 & 684 \\
\hline UG2 & 1206 & 1124 & 689 \\
\hline UG3 & 1208 & 1126 & 688 \\
\hline UG4 & 1217 & 1135 & 695 \\
\hline
\end{tabular}

One of the first attempts to estimate the temperature reached in a cremation pyre from glass artefacts was a study on early Saxon (about 6-7th century) cremation burials in Norfolk (UK). Several glass beads, which were used as grave goods, were recovered that had suffered partial melting. Some of the beads were reheated, and they started to melt at about $850{ }^{\circ} \mathrm{C}$ and were completely liquefied by about $940{ }^{\circ} \mathrm{C}$, suggesting that the cremation temperature was in this range [38]. Glass beads from the archaeological site of Pintia (Padilla de Duero, Valladolid, IV-I centuries) were analysed by Raman spectroscopy in order to detect modification of the glass network structure and to evaluate changes in the polymerisation index. This allowed to estimate the production temperature and the temperature reached during the cremation ritual [39]. However, most cremation burials do not contain partly melted glass objects from which the cremation temperature can be calculated. Therefore, most of the studies of cremation burials evaluated the firing temperature by looking at heat-induced alterations to the hard tissues of the body. Macroscopic changes in the bones occur during burning, resulting in colour change, fragmentation, splitting, and shrinkage [2]. In addition to visual and microscopic observations, Fourier-transform infrared (FTIR) spectroscopy has been used to detect crystallinity changes in bones [40]. The study of fifteen burials from the Roman cemetery of Folly Lane (UK) revealed that the majority of the bone samples were exposed to temperatures over $1000{ }^{\circ} \mathrm{C}$ [41]. During the excavation of three tombs in the cemetery at Kenchreai (Greece), dating from the early to middle Roman periods, several calcined bones were recovered, suggesting that the pyre temperature reached at least $700{ }^{\circ} \mathrm{C}$ [42]. In the burials at the Roman cemetery in Encosta de Sant'Ana in Lisbon (Portugal), bones with a white colouration and heat-induced fractures were found. This colour change indicates that cremation temperatures greater than $645^{\circ} \mathrm{C}$ were achieved $[38,43]$. The research on the human bones from the Roman Pretorio necropolis in Cordoba (Spain) pointed out that about $80 \%$ of the samples were exposed to temperatures higher than $500{ }^{\circ} \mathrm{C}$ [44]. The study of almost 400 cremation tombs from five sites in the Po Valley (northern Italy) dating from the Bronze Age to the Roman period reported the high number of white and light gray bones, proving that temperatures 
between 650 and $900{ }^{\circ} \mathrm{C}$ were obtained during the rituals [45]. Even though these studies take into account the analyses of bones rather than glass, the minimum temperatures reached in these Roman cremation burials are comparable to the temperatures estimated in this study.

\section{Conclusions}

The glass assemblage from cremation deposits at Birdoswald Roman cemetery comprises glass fragments and beads which are representative of three compositional groups: antimony colourless, low-manganese, and mixed antimony and manganese (the majority of the glass fragments). Most of the Roman glass fragments show a transparent blue-green colour and a composition typical of recycled glass. Different colourants were added in the formulation of the coloured, sometimes opaque, beads to impart characteristic hues: cobalt-based compounds for blue glass, while oxidised copper alloys were used in green glass. White calcium antimonate and yellow lead antimonate were employed as opacifiers in blue and green glass, respectively.

The results from this study allow the identification of extreme surface depletion of sodium due to heat exposure during the cremation followed by weathering in the burial environment. Finally, the application of a model of high temperature viscosity of glass has proved to be an effective way to work out the cremation temperature (at least $685^{\circ} \mathrm{C}$ ). The findings of this study play an important role in gaining a better understanding of the funerary rituals, as well as pyre technology in Roman Britain, and demonstrate the limitations of surface analysis on glass assemblages that may have been exposed to high temperatures.

Author Contributions: Conceptualization, methodology, investigation, data curation, writing, review and editing, F.G. All authors have read and agreed to the published version of the manuscript.

Funding: Not applicable.

Institutional Review Board Statement: Not applicable.

Informed Consent Statement: Not applicable.

Data Availability Statement: Not applicable.

Acknowledgments: Sarah Paynter (Historic England) is acknowledged and thanked for her valuable comments on the manuscript. Many thanks also to Nicola Hembrey and Tony Wilmott (Historic England) for context information and specialist advice.

Conflicts of Interest: The author declares that they have no conflict of interest.

\section{References}

1. McKinley, J.I. Chapter 10-In the Heat of the Pyre. In The Analysis of Burned Human Remains, 2nd ed.; Schmidt, C.W., Symes, S.A., Eds.; Academic Press: San Diego, CA, USA, 2015; pp. 181-202.

2. Thompson, T.J.U.; Szigeti, J.; Gowland, R.L.; Witcher, R.E. Death on the frontier: Military cremation practices in the north of Roman Britain. J. Archaeol. Sci. Rep. 2016, 10, 828-836. [CrossRef]

3. Wilmott, T. The Cemetery of the Hadrian's Wall Fort at Birdoswald; Cumberland and Westmorland Antiquarian and Archaeological Society Research Series; Titus Wilson: Kendal, UK, 2022; in preparation.

4. Williams, H. Chapter 15-Towards an Archaeology of Cremation. In The Analysis of Burned Human Remains, 2nd ed.; Schmidt, C.W., Symes, S.A., Eds.; Academic Press: San Diego, CA, USA, 2015; pp. 259-293.

5. McKinley, J.I. A pyre and grave goods in British cremation burials; have we missed something? Antiquity 2015, 68, 132-134. [CrossRef]

6. Brems, D.; Degryse, P.; Hasendoncks, F.; Gimeno, D.; Silvestri, A.; Vassilieva, E.; Luypaers, S.; Honings, J. Western Mediterranean sand deposits as a raw material for Roman glass production. J. Archaeol. Sci. 2012, 39, 2897-2907. [CrossRef]

7. Wedepohl, K.H.; Baumann, A. The use of marine molluskan shells for Roman glass and local raw glass production in the Eifel area (western Germany). Sci. Nat. 2000, 87, 129-132. [CrossRef]

8. Shortland, A.; Schachner, L.; Freestone, I.; Tite, M. Natron as a flux in the early vitreous materials industry: Sources, beginnings and reasons for decline. J. Archaeol. Sci. 2006, 33, 521-530. [CrossRef]

9. Nenna, M.-D.; Vichy, M.; Picon, M. L'atelier de verrier de Lyon, du Ier siècle apr. J.-C, et l'origine des verres «romains». ArchéoSci. Rev. Archéomét. 1997, 21, 81-87. [CrossRef] 
10. Freestone, I.; Gorin-Rosen, Y.; Hughes, M.J. Primary glass from Israel and the production of glass in late antiquity and the early Islamic period. Route Verre 2000, 33, 65-83.

11. Picon, M.; Vichy, M. D'Orient en Occident: L'origine du verre à l'époque romaine et durant le haut Moyen Âge. In Échanges et Commerce du Verre dans le Monde Antique Actes du Colloque de l'Association Française pour l'Archéologie du Verre, 7-9 Juin; Foy, D., Nenna, M.D., Eds.; Editions Mergoil: Aix-en-Provence et Marseille, France, 2003; pp. 17-31.

12. Foy, D.; Vichy, M.; Picon, M. Lingots de verre en Méditerrané occidentale. In Annales du 14e Congrès de l'Association Internationale pour l'Histoire du Verre; AIHV: Amsterdam, The Netherlands, 2000; pp. 51-57.

13. Degryse, P.; Schneider, J. Pliny the Elder and Sr-Nd isotopes: Tracing the provenance of raw materials for Roman glass production. J. Archaeol. Sci. 2008, 35, 1993-2000. [CrossRef]

14. Ganio, M.; Boyen, S.; Fenn, T.; Scott, R.; Vanhoutte, S.; Gimeno, D.; Degryse, P. Roman glass across the Empire: An elemental and isotopic characterization. J. Anal. At. Spectrom. 2012, 27, 743-753. [CrossRef]

15. Price, J.; Cottam, S. Romano-British Glass Vessels: A Handbook; Council for British Archaeology: London, UK, 1998.

16. Jackson, C.M.; Paynter, S. A Great Big Melting Pot: Exploring Patterns of Glass Supply, Consumption and Recycling in Roman Coppergate, York. Archaeometry 2016, 58, 68-95. [CrossRef]

17. Schibille, N.; Sterrett-Krause, A.; Freestone, I. Glass groups, glass supply and recycling in late Roman Carthage. Archaeol. Anthropol. Sci. 2017, 9, 1223-1241. [CrossRef]

18. Boschetti, C.; Leonelli, C. Glass Coloring and Recycling in Late Antiquity: A New Case Study from Aquileia (Italy). J. Glass Stud. 2016, 58, 69-86.

19. Lakatos, T.; Johansson, L.-G.; Simmingsköld, B. Viscosity temperature relations in the glass system $\mathrm{SiO}_{2}-\mathrm{Al}_{2} \mathrm{O}_{3}-\mathrm{Na}_{2} \mathrm{O}-\mathrm{K}_{2} \mathrm{O}-\mathrm{CaO}-$ $\mathrm{MgO}$ in the composition range of technical glasses. Glass Technol. 1972, 13, 88-95.

20. Lakatos, T. Viscosity and temperature relations in glasses composed of $\mathrm{SiO}_{2}-\mathrm{Al}_{2} \mathrm{O}_{3}-\mathrm{Na}_{2} \mathrm{O}-\mathrm{K}_{2} \mathrm{O}-\mathrm{Li} \mathrm{i}_{2} \mathrm{O}-\mathrm{CaO}-\mathrm{MgO}-\mathrm{BaO}-\mathrm{ZnO}-\mathrm{PbO}-$ $\mathrm{B}_{2}$ O. Glastek. Tidskr. 1976, 31, 51-54.

21. Bingham, P.A.; Jackson, C.M. Roman blue-green bottle glass: Chemical-optical analysis and high temperature viscosity modelling. J. Archaeol. Sci. 2008, 35, 302-309. [CrossRef]

22. Henderson, J. The raw materials of early glass production. Oxf. J. Archaeol. 1985, 4, 267-291. [CrossRef]

23. Freestone, I.C.; Leslie, K.A.; Thirlwall, M.; Gorin-Rosen, Y. Strontium Isotopes in the Investigation of Early Glass Production: Byzantine and Early Islamic Glass from the Near East. Archaeometry 2003, 45, 19-32. [CrossRef]

24. Silvestri, A. The coloured glass of Iulia Felix. J. Archaeol. Sci. 2008, 35, 1489-1501. [CrossRef]

25. Henderson, J. Chemical Characterization of Roman Glass Vessels, Enamels and Tesserae. Mater. Res. Soc. Symp. Proc. 2011, 185, 601-607. [CrossRef]

26. Mirti, P.; Casoli, A.; Appolonia, L. Scientific analysis of Roman glass from Augusta Praetoria. Archaeometry 1993, 35, 225-240. [CrossRef]

27. Fredrickx, P.; De Ryck, I.; Janssens, K.; Schryvers, D.; Petit, J.-P.; Döcking, H. EPMA and $\mu$-SRXRF analysis and TEM-based microstructure characterization of a set of Roman glass fragments. Xray Spectrom. 2004, 33, 326-333. [CrossRef]

28. Lahlil, S.; Biron, I.; Galoisy, L.; Morin, G. Rediscovering ancient glass technologies through the examination of opacifier crystals. Appl. Phys. A 2008, 92, 109-116. [CrossRef]

29. Paynter, S.; Kearns, T.; Cool, H.; Chenery, S. Roman coloured glass in the Western provinces: The glass cakes and tesserae from West Clacton in England. J. Archaeol. Sci. 2015, 62, 66-81. [CrossRef]

30. Di Turo, F.; Moro, G.; Artesani, A.; Albertin, F.; Bettuzzi, M.; Cristofori, D.; Moretto, L.M.; Traviglia, A. Chemical analysis and computed tomography of metallic inclusions in Roman glass to unveil ancient coloring methods. Sci. Rep. 2021, $11,11187$. [CrossRef] [PubMed]

31. Paynter, S.; Jackson, C. Clarity and brilliance: Antimony in colourless natron glass explored using Roman glass found in Britain. Archaeol. Anthropol. Sci. 2019, 11, 1533-1551. [CrossRef]

32. Henderson, J.; Warren, S.E. X-ray fluorescence analyses of iron age glass: Beads from Meare and Glastonbury lake villages. Archaeometry 1981, 23, 83-94. [CrossRef]

33. Schiavon, N.; Candeias, A.; Ferreira, T.; Da Conceiçao Lopes, M.; Carneiro, A.; Calligaro, T.; Mirao, J. A combined multianalytical approach for the study of Roman glass from South-West Iberia: Synchrotron $\mu$-XRF, external-Pixe/Pige and BSEM-EDS Archaeometry 2012, 54, 974-996. [CrossRef]

34. Moropoulou, A.; Zacharias, N.; Delegou, E.T.; Maróti, B.; Kasztovszky, Z. Analytical and technological examination of glass tesserae from Hagia Sophia. Microchem. J. 2016, 125, 170-184. [CrossRef]

35. Cailleteau, C.; Weigel, C.; Ledieu, A.; Barboux, P.; Devreux, F. On the effect of glass composition in the dissolution of glasses by water. J. Non-Cryst. Solids 2008, 354, 117-123. [CrossRef]

36. Beerkens, R.G.C. Modeling the Kinetics of Volatilization from Glass Melts. J. Am. Ceram. Soc. 2001, 84, 1952-1960. [CrossRef]

37. Jackson, C.M.; Greenfield, D.; Howie, L.A. An assessment of compositional and morphological changes in model archaeological glasses in an acid burial matrix. Archaeometry 2012, 54, 489-507. [CrossRef]

38. Mays, S. The Archaeology of Human Bones; Routledge: Abingdon, UK, 2010.

39. Pinto, J.; Prieto, A.C.; Coria-Noguera, J.C.; Sanz-Minguez, C.; Souto, J. Investigating glass beads and the funerary rituals of ancient Vaccaei culture (S. IV-I BC) by Raman spectroscopy. J. Raman Spectrosc. 2021, 52, 170-185. [CrossRef] 
40. Thompson, T.J.U.; Islam, M.; Bonniere, M. A new statistical approach for determining the crystallinity of heat-altered bone mineral from FTIR spectra. J. Archaeol. Sci. 2013, 40, 416-422. [CrossRef]

41. Carroll, E.L.; Squires, K.E. The application of quantitative petrography and macroscopic colour change in a comparative analysis of Roman and Anglo-Saxon cremation practices. Int. J. Osteoarchaeol. 2020, 30, 882-890. [CrossRef]

42. Ubelaker, D.; Rife, J. The practice of cremation in the Roman-era cemetery at Kenchreai, Greece. The perspective from archeology and forensic science. Bioarchaeol. Near East 2007, 1, 35-57.

43. Gonçalves, D.; Duarte, C.; Costa, C.; Muralha, J.; Campanacho, V.; Costa, A.; Angelucci, D.E. The Roman cremation burials of the Encosta de Sant' Ana (Lisbon, Portugal). Rev. Port. Arqueol. 2010, 13, 125-144.

44. García-Prósper, E.; Polo-Cerdá, M. Estudio bioantropológico y paleopatológico del sepulcretum de Llanos del Pretorio, Cordoba. In El Sepulcretum de Llanos del Pretorio (Córdoba-España); Vaquerizo, D., Ruiz, A., Rubio, M., Eds.; Edipuglia: Bari, Italy, 2020; pp. 161-172.

45. Masotti, S.; Mongillo, J.; Gualdi-Russo, E. Burned human remains: Diachronic analysis of cremation rituals in necropolises of northern Italy. Archaeol. Anthropol. Sci. 2020, 12, 74. [CrossRef] 